https://helda.helsinki.fi

\title{
Cartan Theorems for Stein Manifolds Over a Discrete Valuation Base
}

\section{Taskinen, Jari}

2019-01

Taskinen , J \& Vilonen , K 2019 , ' Cartan Theorems for Stein Manifolds Over a Discrete

Valuation Base ' , Journal of Geometric Analysis , vol. 29 , no. 1 , pp. 577-615 . https://doi.org/10.1007/s12220-018-C

http://hdl.handle.net/10138/308802

https://doi.org/10.1007/s12220-018-0012-8

unspecified

acceptedVersion

Downloaded from Helda, University of Helsinki institutional repository.

This is an electronic reprint of the original article.

This reprint may differ from the original in pagination and typographic detail.

Please cite the original version. 


\title{
CARTAN THEOREMS FOR STEIN MANIFOLDS OVER A DISCRETE VALUATION BASE
}

\author{
JARI TASKINEN AND KARI VILONEN
}

\section{INTRODUCTION}

In this paper we prove Cartan theorems A and B for Stein manifolds in a relative setting. We work over a base which is a topological discrete valuation ring satisfying certain conditions which we discuss in more detail below.

We were led to this study by questions that arouse in the work of the second author with Kashiwara in the proof of the codimension-three conjecture for holonomic micro differential systems [KaVi]. We could obtain a natural proof of the main results of [KaVi] if we had in our disposal a relative theory of several complex variables where the base is a (certain) topological DVR. In particular, conjecture 1.8 in KaVi - which states that in a relative setting reflexive coherent sheaves extend uniquely across loci of codimension at least three - is a direct analogue of classical results of Trautmann, Siu, and Frisch-Guenot [T, Siu, FG].

Spaces of holomorphic functions with values in a topological vector space have been considered at least since Grothendieck $\mathrm{Gr}$. Extending Cartan theorems A and B to the context of holomorphic functions with values on a locally convex topological vector space was considered in the papers of Bungart [Bu1, and later also by Leiterer. However, they only consider coherent sheaves that come by extension of scalars from ordinary coherent sheaves.

In this paper we consider a different situation. Let $X$ be a complex manifold and $A$ a topological discrete valuation ring. We write $\mathcal{A}_{X}$ for the sheaf of functions on $X$ with values in $A$. Our goal is to prove Cartan theorems $\mathrm{A}$ and $\mathrm{B}$ for coherent $\mathcal{A}_{X}$-modules when $X$ is a Stein manifold. For our methods to work we impose technical conditions on $A$ which are formulated in section 3 , In particular $A$ will be a subring of the formal power series ring $\mathbb{C}[[t]]$ satisfying specific "convergence" conditions. A fundamental example of such convergence conditions, coming up in the context of microdifferential operators, is given in (3.1). We topologize $A$ as a direct limit of Banach algebras, $A=\lim _{h>0} A_{h}$, and one of our basic assumptions is that $A$ is dual nuclear Fréchet. For our arguments to work we have to control the nuclearity of $A$ a bit, as specified in condition (3.4d). Finally, condition (3.4e), which we call subharmonicity, guarantees that we have enough analogues of pseudoconvex domains. In a technical sense it gives us enough plurisubharmonic functions to carry out the $L^{2}$-analysis of Hörmander in our context. It seems reasonable to expect that one obtains a good theory if $A$ is regular

Jari Taskinen was supported in part by the Academy of Finland and the Väisälä Foundation.

Kari Vilonen was supported in part by NSF grants DMS-1402928 \& DMS-1069316, the Academy of Finland, the ARC grant DP150103525, the Humboldt Foundation, and the Simons Foundation. 
local ring satisfying conditions analogous to the ones we pose on discrete valuation rings, but we have not looked into this. In section 2 we give two simple examples which show that Cartan theorems fail when $A$ is not local.

Our main result, (Theorem 8.1) is:

Theorem. Let $A$ be a discrete valuation ring satisfying conditions (3.4) and let $X$ be a Stein manifold. If $\mathcal{F}$ is a coherent $\mathcal{A}_{X}$-module then $\mathrm{H}^{i}(X, \mathcal{F})=0$ for $i \geqslant 1$. Furthermore, the sheaf $\mathcal{F}$ is generated by its global sections.

Our proof of this theorem follows the standard strategy of first proving it for compact Stein domains and then extending it to non-compact ones by a limiting process. However, to carry out this process in our context we have to work in a more general setting where there ring $A$ varies along the complex manifold $X$. This set up is studied in section 4 .

The paper is organized as follows. In section 2 we recall some general old results of Bungart and also give two well-known examples which show that one should not expect the Cartan theorems to hold in great generality. In section 3 we formulate precise conditions on discrete valuation rings which we will be working with. We also show that standard results of several complex variables hold in our setting, in particular, that our structure sheaf is coherent.

In section 4 we introduce the notions of holomorphic functions with values in a varying topological vector space. This is an important technical tool in the proof of the main theorem.

In sections 5 and 6 we prove the Cartan theorems for compact blocks in $\mathbb{C}^{N}$. We do so for structure sheaves which consist of holomorphic functions where the target space varies. To accomplish this we utilize $L^{2}$-techniques of Hörmander. In section 7 we prove approximation lemmas which are used in section 8 where we prove our main result.

\section{Some General Results}

We consider a complex manifold $X$ and the sheaf of holomorphic functions $\mathcal{A}_{X}$ on $X$ with values in a topological ring $A$. Let us write $\mathcal{O}_{X}$ for the sheaf of holomorphic functions on $X$, as usual. Let us recall that the sheaf $\mathcal{O}_{X}$ has a natural structure of sheaf of topological rings if we equip it with the topology uniform convergence on compact sets. Moreover, with this structure $\mathcal{O}_{X}$ is a nuclear Fréchet sheaf, i.e., for $U \subset X$ open the $\mathcal{O}_{X}(U)$ are nuclear Fréchet topological rings. Let us consider a topological ring $A$. We define the structure sheaf $\mathcal{A}_{X}$ as follows. For any open $U$ we set $\mathcal{A}_{X}(U)=A \hat{\otimes} \mathcal{O}_{X}(U)$; here and in the rest of the paper all topological tensor products are projective tensor products. The sections $\mathcal{A}_{X}(U)$ can also be identified with holomorphic functions on $U$ with values in $A$ which we equip with the topology uniform convergence on compact sets.

Cartan theorems A and B were studied by Bungart for sheaves that arise from ordinary coherent sheaves on $X$ by extending scalars. He obtained the following results:

Theorem 2.1. (Bungart, Bu1/Theorem B in section 11]) Let $\mathcal{F}$ be a coherent analytic sheaf on a Stein space $X$. Then for every Frechet space $E$ we have $H^{q}(X, E \hat{\otimes} \mathcal{F})=0$ for $q>0$. 
Theorem 2.2. (Bungart, Bu1]/Theorem $B^{*}$ in section 17]) Let $\mathcal{F}$ be a coherent analytic sheaf on the Stein space $X$ and $K$ a holomorphically convex compact subset of $X$, and $E$ is any (quasi-)complete locally convex space. Then $H^{q}(K, E \hat{\otimes} \mathcal{F})=0$ for $q>0$.

Theorem 2.3. (Bungart, Bu1][Theorem $A$ in section 17]) Let $X$ be Stein space, $\mathcal{F}$ is a coherent sheaf of $\mathcal{O}_{X}$-modules on $X$, and $E$ is any (quasi-) complete locally convex space. Then $E \hat{\otimes} \mathcal{F}$ is generated by global sections.

The first theorem 2.1 can be proved by a straightforward extensions of scalars argument. To explain it, let us recall the following:

Lemma 2.4. Let $F$ be a locally convex space and

$$
0 \rightarrow E_{1} \stackrel{\alpha}{\rightarrow} E_{2} \stackrel{\beta}{\rightarrow} E_{3} \rightarrow 0
$$

a topologically exact sequence of locally convex spaces. Assume that either $F$ or $E_{2}$ is nuclear and that $F$ and $E_{2}$ are both Frechet spaces or both DF spaces. Then the sequence

$$
0 \rightarrow F \hat{\otimes} E_{1} \stackrel{1 \hat{\otimes} \alpha}{\longrightarrow} F \hat{\otimes} E_{2} \stackrel{1 \hat{\otimes} \beta}{\longrightarrow} F \hat{\otimes} E_{3} \rightarrow 0
$$

is exact.

Let us choose a Stein cover $\mathcal{U}$ of $X$ and form the Chech complex $C^{\cdot}(\mathcal{U}, \mathcal{F})$. As the topological vector spaces in $C^{\cdot}(\mathcal{U}, \mathcal{F})$ are nuclear Frechet the lemma implies that tensoring with $E$ commutes with taking cohomology and thus we obtain theorem 2.1 .

Our interest lies in finding a good class of topological algebras $A$ so that the Cartan theorems hold for coherent $\mathcal{A}_{X}$-modules. We present two standard examples which illustrate that one has to exercise some caution if one is to generalize these theorems for coherent sheaves of $\mathcal{A}_{X}$-modules even for some rather reasonable $A$.

2.1. Example 1. Let us take $A=\mathbb{C}[t]$ and $X=\mathbb{C}$. We equip $A$ with its direct limit topology induced by finite dimensional subspaces. Note that the sheaf $\mathcal{A}_{\mathbb{C}}$ is coherent. We will consider the following exact sequence:

$$
0 \rightarrow \mathcal{J}_{\mathbb{Z}} \rightarrow \mathcal{O}_{\mathbb{C}} \rightarrow \mathcal{O}_{\mathbb{Z}} \rightarrow 0
$$

where we consider $\mathbb{Z} \subset \mathbb{C}$ as a sub variety. We can tensor this sequence with $A$ to obtain an exact sequence

$$
0 \rightarrow \mathcal{J}_{\mathbb{Z}}^{A} \rightarrow \mathcal{A}_{\mathbb{C}} \rightarrow \mathcal{A}_{\mathbb{Z}} \rightarrow 0
$$

where we have written, as before, $\mathcal{A}_{Y}$ for the sheaf of holomorphic functions with values in $Y$. Now we see that on the level of global sections the last two terms become

$$
\Gamma\left(\mathbb{C}, \mathcal{A}_{\mathbb{C}}\right)=\left\{\sum_{i=0}^{m} f_{i} t^{i} \mid f_{i} \in \mathcal{O}_{\mathbb{C}}(\mathbb{C})\right\} \rightarrow\left\{\left(n, \sum_{i=0}^{m_{n}} a_{i, n} t^{i}\right)\right\}=\Gamma\left(\mathbb{C}, \mathcal{A}_{\mathbb{Z}}\right)
$$

and the map is given by

It is clear that the element

$$
\sum_{i=0}^{m} f_{i} t^{i} \mapsto\left(n, \sum_{i=0}^{m_{n}} f_{i}(n) t^{i}\right)
$$

$$
\left(n, t^{n}\right)
$$


cannot come from any $\sum_{i=0}^{m} f_{i} t^{i}$. Thus, from the exact sequence

$$
0 \rightarrow \Gamma\left(\mathbb{C}, \mathcal{A}_{\mathbb{Z}}\right) \rightarrow \Gamma\left(\mathbb{C}, \mathcal{A}_{\mathbb{C}}\right) \rightarrow \Gamma\left(\mathbb{C}, \mathcal{A}_{\mathbb{Z}}\right) \rightarrow \mathrm{H}^{1}\left(\mathbb{C}, \mathcal{A}_{\mathbb{Z}}\right) \rightarrow \ldots
$$

we conclude that

$$
\mathrm{H}^{1}\left(\mathbb{C}, \mathcal{J}_{\mathbb{Z}}^{A}\right) \neq 0,
$$

although $\mathcal{J}_{\mathbb{Z}}^{A}$ is coherent. Thus, Cartan's theorem B fails in this case.

2.2. Example 2. Let us now take as our $A$ the $\operatorname{ring} A=\mathbb{C}\left[t, t^{-1}\right]$ and for our $X$ we take $X=\mathbb{C} / \mathbb{Z}$. Again we equip $A$ with its direct limit topology induced by finite dimensional subspaces. We consider the constant sheaf $\mathcal{A}_{\mathbb{C}}$ on $\mathbb{C}$ and consider the $\mathbb{Z}$ action on it via $(n \cdot f)(t)=t^{n} f(t-n)$. Viewed in this manner $\mathcal{A}_{\mathbb{C}}$ is a $\mathbb{Z}$-equivariant sheaf on $\mathbb{C}$ and so it induces a sheaf $\mathcal{A}_{\mathbb{C}} / \mathbb{Z}$ on $X=\mathbb{C} / \mathbb{Z}$. This sheaf is clearly locally free, so it is coherent. We now consider

$$
\Gamma\left(\mathbb{C} / \mathbb{Z}, \mathcal{A}_{\mathbb{C}} / \mathbb{Z}\right)=\Gamma\left(\mathbb{C}, \mathcal{A}_{\mathbb{C}}\right)^{\mathbb{Z}}
$$

Clearly,

$$
\Gamma\left(\mathbb{C}, \mathcal{A}_{\mathbb{C}}\right)^{\mathbb{Z}}=0 .
$$

Thus, Cartan's theorem A fails as the sheaf is not generated by its global sections because there are not any.

\section{OUR SET UP}

In this section we explain the conditions we will be imposing on the topological ring $A$. We continue to consider a complex manifold $X$ and the sheaf of holomorphic functions $\mathcal{A}_{X}$ on $X$ with values in a topological ring $A$ as was explained in the previous section.

From the point of view of $\mathrm{KaVi}$ the ring of interest is the following regular local ring $A$. Consider the formal power series ring $\widehat{A}=\mathbb{C}[[t]]$. It is a discrete valuation ring. We define a subring $A$ of $\widehat{A}$ in the following manner. For any $h>0$ we define a norm \|\|$_{h}$ on $\widehat{A}$ by the formula

$$
\left\|\sum_{j=0}^{\infty} a_{j} t^{j}\right\|_{h}=\sum_{j=0}^{\infty}\left|a_{j}\right| \frac{h^{j}}{j !} .
$$

We write $A_{h}$ for the subring consisting of elements $a$ of $\widehat{A}$ with $\|a\|_{h}<\infty$. The ring $A_{h}$ is a Banach local ring as is not so difficult to see. Finally, we set

$$
A=\underset{h \rightarrow 0}{\lim _{h}} A_{h} .
$$

The topological ring $A$ is a dual nuclear Fréchet discrete valuation ring, a DNF DVR.

We will next define a class of topological rings that we will be working with which includes the example discussed above. First of all, we need to impose reasonable conditions on $A$ so that $\mathcal{A}_{X}$ is coherent and so that the stalks $\mathcal{A}_{X, x}$ are regular local rings. Thus, we have to assume that $A$ is a regular local ring. In light of Example 1 of section 2 this is a reasonable assumption anyway. Let us write $\mathfrak{m}$ for the maximal ideal in 
$A$ and let us assume that the dimension of $A$ is $r$. We complete $A$ with respect to $\mathfrak{m}$ to obtain a complete local ring $\hat{A} \cong \mathbb{C}\left[\left[t_{1}, \ldots, t_{r}\right]\right]$. Then

$$
\mathbb{C}\left[t_{1}, \ldots, t_{r}\right] \subset A \subset \hat{A} \cong \mathbb{C}\left[\left[t_{1}, \ldots, t_{r}\right]\right] .
$$

Hence $A$ can be viewed as consisting of power series in $r$ variables satisfying some kind of a "convergence" condition. The main results in this paper should hold for regular local rings of any dimension. However, we will make the further assumption that $\operatorname{dim} A=1$, i.e., that $A$ is a DVR. As $A \subset \mathbb{C}[[t]]$, any element $f \in A$ can be written as

$$
f=\sum_{j=0}^{\infty} a_{j} t^{j}
$$

We can thus view the $A$ as providing us with a (usually very small!) neighborhood of the origin in $\mathbb{C}$.

We assume that the topology on $A$ is given as a direct limit of Banach algebras, i.e., that

$$
A=\underset{h>0}{\lim _{h}} A_{h}
$$

where the $A_{h}$, with $0<h<S$ for some fixed $S \in \mathbb{R}^{+}$, are commutative Banach algebras with norm \|\|$_{h}$ and we will assume furthermore that the maps $A_{h} \rightarrow A_{k}$, for $h>k>0$ are nuclear ring homomorphisms which we can assume to be inclusions.

We choose the norms \|\|$_{h}$ in such a way that

$$
\left\|\sum_{j=0}^{\infty} a_{j} t^{j}\right\|_{h}=\sum_{j=0}^{\infty}\left|a_{j}\right|\left\|t^{j}\right\|_{h} .
$$

(We remark that this assumption is not as restrictive as it might appear: since we will anyway assume that $A$ is a dual nuclear Fréchet space, see below, assuming in addition only that $\left\{t^{i} \mid i=0,1, \ldots\right\}$ is a Schauder basis for $A$ would imply that the norms (3.3) give $A$ its own topology, see [P Theorems 10.1.2 and 10.1.4.]. Furthermore, the Schauder basis property follows by just assuming that the (unique) representation (3.2) converges in the topology of $A$ for every $f$.)

For the norms (3.3) to give us a Banach algebra it is necessary and sufficient that

$$
\left\|t^{j+l}\right\|_{h} \leqslant\left\|t^{j}\right\|_{h}\left\|t^{l}\right\|_{h} \quad \text { for all } j, l
$$

as is easy to show. The Banach algebra $A_{h}$ then consists of $\sum_{j=0}^{\infty} a_{j} t^{j}$ such that $\left\|\sum_{j=0}^{\infty} a_{j} t^{j}\right\|_{h}=\sum_{j=0}^{\infty}\left|a_{j}\right|\left\|t^{j}\right\|_{h}$ is finite.

The simplest such an $A$ is given by germs of holomorphic functions at the origin. In that case

$$
\left\|\sum_{j=0}^{\infty} a_{j} t^{j}\right\|_{h}=\sum_{j=0}^{\infty}\left|a_{j}\right| h^{j} \quad \text { and then }\left\|t^{j}\right\|_{h}=h^{j} .
$$

For any $h$ we can also form the following two topological rings:

$$
A_{\breve{h}}=\underset{k>h}{\lim _{k}} A_{k}
$$


and

$$
A_{\widehat{h}}=\lim _{h<k} A_{k}
$$

As we have assumed that the maps $A_{h} \rightarrow A_{k}$, for $h>k>0$ are nuclear ring homomorphisms we see that $A_{\breve{h}}$ is a DNF (dual nuclear Fréchet ) ring and the $A_{\widehat{h}}$ is a NF (nuclear Fréchet ) ring. In our example of germs of holomorphic functions the ring $A_{\breve{h}}$ is the ring of holomorphic functions on the (compact) closed disk or radius $h$ and the $\operatorname{ring} A_{\widehat{h}}$ is the ring of holomorphic functions on the open disk or radius $h$. The $A_{\breve{h}}$ and $A_{\widehat{h}}$ are the analogues of these familiar constructions and the ring $A_{\breve{h}}$ will play an important role in the rest of the paper.

3.1. The assumptions on the families $A_{h}$. We will now formulate precise conditions on the $\left\|t^{j}\right\|_{h}$ which will be in force for the rest of the paper. We will first list all the conditions and then explain their meaning. To that end let us write

$$
R(h, j):=\frac{\left\|t^{j+1}\right\|_{h}}{\left\|t^{j}\right\|_{h}} .
$$

and

$$
N_{j}:[0, S] \rightarrow \mathbb{R}^{+}, \quad N_{j}(h):=\left\|t^{j}\right\|_{h}^{2}
$$

for every $j \in \mathbb{N}$.

First of all, in order for the $A_{h}$ to be Banach algebras we require

$$
\left\|t^{j+l}\right\|_{h} \leqslant\left\|t^{j}\right\|_{h}\left\|t^{l}\right\|_{h} \quad \text { for all } j, l . \quad \text { (Banach algebra) }
$$

For the purposes of the arguments we furthermore normalize things so that

$$
\left\|t^{j}\right\|_{h} \leqslant 1 \text { and } R(h, j) \leqslant 1 \text { for all } j \in \mathbb{N} . \quad \text { (normalization) }
$$

In particular, the sequence $\left\|t^{j}\right\|_{h}$ is decreasing.

We also assume that

$$
R(h, j) \rightarrow 0 \text { as } j \rightarrow \infty . \quad \text { (locality) }
$$

This condition implies that the $A_{h}$ are local rings: the numbers $\left\|t^{j}\right\|_{h}$ have to decay fast enough, faster than exponentially, as $j \rightarrow \infty$. We could relax this condition a bit, but it simplifies the discussion to pose it. Basically it only excludes the classical case of germs of holomorphic functions.

The first crucial assumption is the following: for every pair $h<k$ there exists a constant $K_{h, k}>0$ such that for every $j$,

$$
\left\|t^{j}\right\|_{h} \leqslant K_{h, k} \min \left\{j^{-1}, R(k, j)\right\}\left\|t^{j}\right\|_{k} . \quad \text { (controlled nuclearity) }
$$

In particular, we are assuming $\left\|t^{j}\right\|_{h} \rightarrow 0$ as $h \rightarrow 0$, for every $j$.

Finally, as a second crucial assumption we assume that every $N_{j}$ is two times continuously differentiable, decreasing, and that

$$
-\frac{d^{2}}{d h^{2}} \log N_{j}(h) \geqslant \frac{1}{h} \frac{d}{d h} \log N_{j}(h) \quad \text { (subharmonicity) }
$$

for all (small enough) $h \in(0, S]$ and for all $j$.

The first three conditions are rather straightforward. We will discuss briefly the meaning of the last two conditions. 
Let us unravel the meaning of condition (3.4d d . It is a "controlled" nuclearity requirement. More precisely, considering the inequality with the $j^{-1}$ term guarantees nuclearity of the maps $A_{h} \rightarrow A_{k}$. Moreover it puts a bound on the nuclearity which we will make crucial use of in section 6 where we have to pass between our norms and $L^{2}$-norms. Considering the inequality with the $R(C, j)$ term gives us the following inequality:

$$
\left\|t^{j}\right\|_{h} \leqslant K_{h, k}\left\|t^{j+1}\right\|_{k} .
$$

This condition is needed in order to prove lemma 3.1 , i.e., that $A_{\breve{h}}$ is a DVR. It expresses some form of nuclearity in families. Therefore we call condition (3.4d) "controlled" nuclearity .

Finally, condition (3.4e) guarantees that we have enough analogues of pseudoconvex domains. In the classical setting Stein submanifolds have a cofinal family of neighborhoods which are Stein. In our setting we need an analogue of this statement. To have such cofinal families we have to impose condition (3.4e). In a technical sense it gives us enough plurisubharmonic functions to carry out the $L^{2}$-analysis of Hörmander in our setting. This is done is section 6 .

\section{Examples of functions satisfying all conditions.}

$$
\begin{aligned}
& \text { (i) } \frac{1}{j !} h^{j^{\gamma}}, \gamma \geqslant 1, \\
& \text { (ii) } j^{-k} h^{j^{\gamma}}, k=1,2, \ldots, \gamma>1, h \text { small enough, } \\
& \text { (iii) } e^{-j^{k}} h^{j^{\gamma}}, k=1,2, \ldots, \gamma>k, \\
& \text { (iv) } \frac{1}{j !} e^{-\gamma j / h}, \gamma \geqslant 1 \\
& \text { (v) } \frac{1}{j !} e^{\left(1-\gamma^{j}\right) / h}, \gamma \geqslant 2 .
\end{aligned}
$$

In the rest of the section we will prove basic facts about the sheaves $\mathcal{A}_{X}$. In this section we do not make use of the subharmonicity condition (3.4e). We start with:

Lemma 3.1. The ring $A_{\breve{h}}$ is a $D V R$

Proof. First, the condition (3.4c) implies that the rings $A_{h}$ are local ring with maximal ideal $\mathfrak{m}_{h}=\left\{\sum_{j=0}^{\infty} a_{j} t^{j} \in A_{h} \mid a_{0}=0\right\}$. Hence, $A_{\breve{h}}$ is a local ring with maximal ideal $\check{\mathfrak{m}}_{h}=\left\{\sum_{j=0}^{\infty} a_{j} t^{j} \in A_{\breve{h}} \mid a_{0}=0\right\}$. It suffices to show that $\mathfrak{m}_{h}=(t)$. Let $f \in \check{\mathfrak{m}}_{h}$. Then there is a $k>h$ such that $f \in A_{k}$ and of course $f(0)=0$. Let us write $f(t)=\operatorname{tg}(t)$ with $g(t)=\sum_{j=0}^{\infty} a_{j} t^{j-1} \in \hat{A}$. We will show that $g(t) \in A_{l}$ for any $l$ such that $k>l>h$. As $f \in A_{k}$ we see that

$$
\|f\|_{k}=\left\|\sum_{j=0}^{\infty} a_{j} t^{j}\right\|_{k}=\sum_{j=0}^{\infty}\left|a_{j}\right|\left\|t^{j}\right\|_{k}<\infty .
$$

Let us now choose any $l$ such that $h<l<k$. To show that $\|g(t)\|_{l}<\infty$ we consider:

$$
\|g(t)\|_{l}=\sum_{j=0}^{\infty}\left|a_{j}\right|\left\|t^{j-1}\right\|_{l}
$$


Comparing the series (3.7) to the series (3.6) and using the condition (3.4d) in the form of (3.5) we conclude that $g(t) \in A_{l}$.

As the spectrum of $A_{h}$ then consists of the origin only we see by the spectral radius formula that

$$
\left\|t^{n}\right\|_{h}=\left(\epsilon_{n}\right)^{n} \quad \text { where } \quad \lim _{n \rightarrow \infty} \epsilon_{n}=0 .
$$

Remark 3.2. In the case of our motivating example (3.1) we see that the $\epsilon_{n}$ is essentially proportional to $\frac{1}{n}$ by the Stirling formula.

Let us recall that we have assumed that $\|t\|_{h} \leqslant 1$. Then $\left\|t^{n}\right\|_{h} \leqslant\left\|t^{n-1}\right\|_{h}\|t\|_{h} \leqslant$ $\left\|t^{n-1}\right\|_{h} \leqslant 1$. Now,

$$
\epsilon_{n}=\left(\left\|t^{n}\right\|_{h}\right)^{\frac{1}{n}} \leqslant\left(\left\|t^{n-1}\right\|_{h}\right)^{\frac{1}{n}}=\left(\left\|t^{n-1}\right\|_{h}\right)^{\frac{1}{n-1}}\left(\left\|t^{n-1}\right\|_{h}\right)^{\frac{n-1}{n}} \leqslant \epsilon_{n-1} .
$$

Thus, we conclude that

The sequence $\epsilon_{n}$ is decreasing.

Let us now come back to analyze the rings $\mathcal{A}_{X}$. We write $\mathcal{A}_{X}^{h}=A_{h} \hat{\otimes} \mathcal{O}_{X}$ for the sheaf of holomorphic functions with values in $A_{h}$ and we write $\mathcal{A}_{X}^{\breve{h}}=A_{\breve{h}} \hat{\otimes} \mathcal{O}_{X}$ for the sheaf of holomorphic functions with values in $A_{\breve{h}}$.

Lemma 3.3. The stalks $\mathcal{A}_{X, x}$ and $\mathcal{A}_{X, x}^{\check{h}}$ are local rings.

Proof. The argument is the same in both cases, so we work with $\mathcal{A}_{X, x}$. The maximal ideal $\mathfrak{m}_{A, x} \subset \mathcal{A}_{X, x}$ consists of functions $f \in \mathcal{A}_{X, x}$ such that $f(x) \in \mathfrak{m}$. To prove that $\mathcal{A}_{X, x}$ is local we have to show that any $f \notin \mathfrak{m}_{A, x}$ is invertible. Any element $f \in \mathcal{A}_{X, x}$ is represented by a series

$$
f=\sum a_{\alpha} x^{\alpha} \quad a_{\alpha} \in A ;
$$

here we have replaced $X$ by $\mathbb{C}^{n}$ and assumed that $x$ is the origin. This series converges in some neighborhood $U$ of the origin. Let us now restrict $f$ to a smaller neighborhood $V$ such that $\bar{V} \subset U$. As the ring $A$ is equipped with a direct limit topology, there is an $h$ such that $\left.f\right|_{\bar{V}} \in \mathcal{A}_{h}(\bar{V})$. Note also that if $f \notin \mathfrak{m}_{A, x}$ then the first term $a_{0} \in A^{*}=A-\mathfrak{m}$, the units in $A$. Thus,

$$
a_{0}^{-1} f=1+\sum a_{\alpha} x^{\alpha}
$$

Now, as $\sum a_{\alpha} x^{\alpha}$ vanishes at the origin, we can, by making the neighborhood $U$ smaller if necessary, assume that $\left\|\sum a_{\alpha} x^{\alpha}\right\|_{h}<1$. Thus $a_{0}^{-1} f$ and hence $f$ is invertible.

Remark 3.4. The sheaves $\mathcal{A}_{X}$ and $\mathcal{A}_{X}^{\check{h}}$ are defined in the same way. For emphasis we will make statements in both cases, but of course for the proof we can just think in terms of $\mathcal{A}_{X}$.

Theorem 3.5. The stalks $\mathcal{A}_{X, x}$ and $\mathcal{A}_{X, x}^{\check{h}}$ are regular local rings. 
To prove the theorem we work locally so that we can assume that $X=\mathbb{C}^{n}$ and we choose local coordinates $x_{1}, \ldots, x_{n}$ on $\mathbb{C}^{n}$ such that the point $x$ corresponds to the origin. We write $f$ in local coordinates

$$
f=\sum a_{i} t^{i} \quad a_{i} \in \mathcal{O}_{X, x}
$$

We prove this statement in the standard manner by first proving an appropriate Weierstrass division theorem. We follow the classical argument as presented in GrRe, Chapter 2]. We will also try to stick to the notation there as closely as possible. As a first step we argue that by a change of coordinates we can write $f$ so that it is $t$-regular, i.e., that there is a $b$ such that

$$
a_{0}(0)=\cdots=a_{b-1}(0)=0 \text { and } a_{b}(0) \neq 0 .
$$

As we work locally, the function $f$ is holomorphic on some closed polydisk of radius $\rho=\left(\rho_{1}, \ldots, \rho_{n}\right)$ and on that polydisk $f \in \mathcal{A}_{X}^{h}$ for some $h$. Thus we can consider the following norm \|\|$_{\rho}$ on $f$ by

$$
\|f\|_{\rho}=\sum_{i, \alpha}\left|a_{\alpha, i}\right| \rho^{\alpha}\left\|t^{i}\right\|_{h} \quad \text { where } \quad a_{i}=\sum a_{\alpha, i} x_{1}^{\alpha_{1}} \ldots x_{n}^{\alpha_{n}} \quad \rho^{\alpha}=\rho_{1}^{\alpha_{1}} \ldots \rho_{n}^{\alpha_{n}} .
$$

We now substitute

$$
w_{1}=x_{1}+c_{1} t, \ldots, w_{n}=x_{n}+c_{n} t
$$

to get the new coordinates $w_{1}, \ldots, w_{n}, t$. A generic choice of small such $c_{i}$ will make $f$ regular in $t$. It is perhaps good to note that outside of special cases we can only make $f$ regular in $t$ and not in any of the other variables.

We now write $f$ in this new set of variables as

$$
f=\sum_{i, \alpha} b_{\alpha, i} w_{1}^{\alpha_{1}} \ldots w_{n}^{\alpha_{n}} t^{i}=\sum_{i} b_{i} t^{i} .
$$

In these new coordinates we of course will get a different $\rho$ for the radius of convergence. However, it is important that we do not change $h$.

We perform this substitution one variable at a time and keep the other variables fixed. So, we are reduced in the one variable situation in the base and we write $x=x_{1}$, $w=w_{1}=x+c t$ and the $\rho=\rho_{1}$. Our $f$ can now be written as

$$
f=\sum_{i, j} a_{i, j} x^{j} t^{i}
$$

and after the substitution

$$
\begin{gathered}
f=\sum_{i, j} a_{i, j}(w+c t)^{j} t^{i}=\sum_{i, j} a_{i, j} \sum_{p+q=j}\left(\begin{array}{c}
p+q \\
p
\end{array}\right) w^{p} c^{q} t^{i+q}= \\
=\sum_{i, p, q} a_{i, p+q}\left(\begin{array}{c}
p+q \\
p
\end{array}\right) w^{p} c^{q} t^{i+q} .
\end{gathered}
$$

We now consider (3.12) and we obtain

$$
\|f\|_{\rho}=\sum_{i, j}\left|a_{i, j}\right| \rho^{j} \epsilon_{i}^{i}<\infty
$$


We then write

$$
\lambda_{i j}=\left|a_{i, j}\right| \rho^{j} \epsilon_{i}^{i} \text { and then }\left|a_{i, j}\right|=\lambda_{i j} \rho^{-j} \epsilon_{i}^{-i} \text { and } \sum_{i, j} \lambda_{i j}<\infty .
$$

We furthermore set

$$
\lambda_{i}=\max \left\{\lambda_{i j} \mid j=0, \ldots\right\} \text { and we still have } \sum \lambda_{i}<\infty .
$$

This allows us to estimate the norm in (3.13) after the substitution

$$
\begin{gathered}
\|f\|_{r}=\sum_{i, p, q}\left|a_{i, p+q}\right|\left(\begin{array}{c}
p+q \\
p
\end{array}\right) r^{p} c^{q}\|t\|_{h}^{i+q}= \\
\sum_{i, p, q} \lambda_{i, p+q}\left(\begin{array}{c}
p+q \\
p
\end{array}\right) \rho^{-p-q} \epsilon_{i}^{-i} r^{p} c^{q} \epsilon_{i+q}^{i+q} \leqslant \\
\sum_{i, q} \lambda_{i}\left(\frac{\epsilon_{i+q}}{\epsilon_{i}}\right)^{i}\left(\frac{c \epsilon_{i+q}}{\rho}\right)^{q} \sum_{p}\left(\begin{array}{c}
p+q \\
p
\end{array}\right)\left(\frac{r}{\rho}\right)^{p}= \\
=\sum_{i, q} \lambda_{i}\left(\frac{\epsilon_{i+q}}{\epsilon_{i}}\right)^{i}\left(\frac{c \epsilon_{i+q}}{\rho}\right)^{q}\left(1-\frac{r}{\rho}\right)^{-q-1} \\
=\left(1-\frac{r}{\rho}\right)^{-1} \sum_{i, q} \lambda_{i}\left(\frac{\epsilon_{i+q}}{\epsilon_{i}}\right)^{i}\left(\frac{c \epsilon_{i+q}}{\rho-r}\right)^{q}<\infty
\end{gathered}
$$

because by (3.9) we have $\epsilon_{i+q} \leqslant \epsilon_{i}$, by (3.8) we have $\epsilon_{i+q} \rightarrow 0$ when $i+q \rightarrow \infty$, and by (3.14) we have $\sum \lambda_{i}<\infty$.

We proceed in this manner one variable at a time keeping the others constant. We thus have reached the following conclusion:

Given $f \in \mathcal{A}_{X, x}^{h}$ we can make it $t$-regular by a coordinate change (3.11).

Let us consider $f \in \mathcal{A}_{X, x}$. The function $f$ is holomorphic on some closed polydisk of radius $\rho=\left(\rho_{1}, \ldots, \rho_{n}\right)$ and on that polydisk $f \in \mathcal{A}_{X}^{h}$ for some $h$. We consider the norm \|\|$_{\rho}$ defined in (3.10). As before, we write

$$
f=\sum a_{i} t^{i} \quad a_{i} \in \mathcal{O}_{X, x} .
$$

and then we write

$$
\hat{f}=\sum_{0}^{b-1} a_{i} t^{i} \quad \tilde{f}=\sum_{b}^{\infty} a_{i} t^{i-b} \quad f=\hat{f}+\tilde{f} t^{b} .
$$

Note that although, of course, $\tilde{f} t^{b} \in \mathcal{A}_{X, x}^{h}$, the function $\tilde{f}$ does not necessarily lie in $\mathcal{A}_{X, x}^{h}$. However, it does lie in any $\mathcal{A}_{X, x}^{k}$ for $k<h$. We see this by applying the following general principle inductively:

Lemma 3.6. Let $g \in \mathcal{A}_{X}^{\check{h}^{\prime}}(U)$ and assume that $g(x) \in \mathfrak{m}_{h^{\prime}}$ for all $x \in U$. Then have $g=t \tilde{g}$ with $\tilde{g} \in \mathcal{A}_{X}^{\check{h}^{\prime}}(U)$. 
Proof. Let us then consider the map $t: A_{\breve{h^{\prime}}} \rightarrow A_{\breve{h}^{\prime}}$ given by multiplication by $t$. By our hypotheses the map $t: A_{\breve{h}^{\prime}} \rightarrow \check{\mathfrak{m}}_{h^{\prime}}$ is continuous and a bijection. As both $A_{\breve{h}^{\prime}}$ and $\check{\mathfrak{m}}_{h^{\prime}}$ are DNF, the open mapping theorem applies and hence $t: A_{\breve{h}^{\prime}} \rightarrow \mathfrak{m}_{h^{\prime}}$ is an isomorphism. Thus, $t^{-1}: \check{\mathfrak{m}}_{h^{\prime}} \rightarrow A_{\breve{h}^{\prime}}$ is a well defined continuous map and so we can write $g=t \tilde{g}$ where $\tilde{g} \in \mathcal{A}_{X}^{\breve{h^{\prime}}}(U)$.

We apply this lemma after first passing to $\mathcal{A}_{X, x}^{\breve{h}^{\prime}}$ with $k<h^{\prime}<h$ and repeat the process to extract the $b$ copies of $t$ from $\tilde{f} t^{b}$. We now also have

$$
\|\hat{f}\|_{\rho} \leqslant\|f\|_{\rho} \quad\|\tilde{f}\|_{\rho} \leqslant\|f\|_{\rho}\left\|t^{b}\right\|^{-1} .
$$

Recall that any statements like this we make about norms are valid as long as both sides are defined. In particular, the latter inequality holds for any $t$-norm $k$ where $k<h$.

We are now ready to state and prove the Weierstrass division theorem:

Proposition 3.7. If $g \in \mathcal{A}_{X, x}$ has order $b$ in $t$ then for any $f \in \mathcal{A}_{X, x}$ there exists $a$ $q \in \mathcal{A}_{X, x}$ and an $r \in \mathcal{O}_{X, x}[t]$ with degree of $r$ in $t$ less than $b$ such that $f=q g+r$.

Proof. To prove this result, we proceed just as in the classical case. The argument in GrRe, Chapter 2, §1] can be adopted to our situation and we briefly indicate the necessary changes trying to stick as close to the notation there as possible. First of all, we can view the stalk $\mathcal{A}_{X, x}$ as a direct limit of Banach algebras $B_{\rho}^{h}$, where $\rho=\left(r_{1}, \ldots, r_{n}\right)$ is a sequence of positive real numbers and the norms are given by

$$
\text { For } f=\sum b_{\alpha} x^{\alpha} \text { we set }\|f\|_{h, \rho}=\sum\left\|b_{\alpha}\right\|_{h} r_{1}^{\alpha_{1}} \ldots r_{n}^{\alpha_{n}}
$$

just as in GrRe, Chapter $2, \S 1$ ] for the case $A_{k}=\mathbb{C}$ and as we have done above. Note that because $g$ is $b$-regular in $t$ then $\tilde{g}$ is invertible. We first note that we can choose $\rho$ sufficiently small so that $g, \hat{g}, \tilde{g}$, and $\tilde{g}^{-1}$ lie in $B_{\rho}^{h}$ for some $h$. We will further adjust $h$ and $\rho$ so that we also have $f \in B_{\rho}^{h}$. Note that

$$
\left\|t^{b}-g \tilde{g}^{-1}\right\|=\left\|\hat{g} \tilde{g}^{-1}\right\| \leqslant\|\hat{g}\|\left\|\tilde{g}^{-1}\right\|
$$

Now, we have for $g=\sum a_{i} t^{i}$ that $a_{0}(0)=\cdots=a_{b-1}(0)=0$ and hence $\|\hat{g}\|$ can be made arbitrarily small by shrinking $\rho$. As $\tilde{g}^{-1}$ is invertible, its norm can be bounded away from zero. Thus, for any $\epsilon>0$ we can, by shrinking $\rho$, arrange things so that

$$
\left\|t^{b}-g \tilde{g}^{-1}\right\|<\epsilon\left\|t^{b}\right\|
$$

We now set

$$
v_{0}=f, \ldots, v_{j+1}=\left(t^{b}-g \tilde{g}^{-1}\right) \tilde{v}_{j}=-\hat{g} \tilde{g}^{-1} \tilde{v}_{j}, \ldots
$$

By (3.16) we get that

$$
\left\|\tilde{v}_{j}\right\|_{\rho} \leqslant\left\|v_{j}\right\|_{\rho}\left\|t^{b}\right\|^{-1} \quad \text { and then } \quad\left\|v_{j+1}\right\| \leqslant \epsilon\left\|v_{j}\right\|_{\rho} .
$$

Recall that for a given $h$ the estimate (3.16) holds for any $k<h$ but not necessarily for $h$ itself. We make use of the estimate every time we form a $\tilde{v}_{j}$ but for any $k$ we can 
choose $h>k_{0}>k_{1}>\cdots>k_{j}>\cdots>k$. When we form $\tilde{v}_{j}$ we pass from $k_{j-1}$ to $k_{j}$. Thus, the estimates above holds for any $k<h$. We now set, for $k<k^{\prime}<h$

$$
v=\sum_{0}^{\infty} v_{j} \in B_{\rho}^{k^{\prime}} \quad q=\tilde{g}^{-1} \tilde{v} \in B_{\rho}^{k} \quad r=\hat{v} \in B_{\rho}^{k} .
$$

Then

$$
f=\sum_{0}^{\infty}\left(v_{j}-v_{j+1}\right)=\sum_{0}^{\infty}\left(g \tilde{g}^{-1} \tilde{v}_{j}+\tilde{v}_{j}\right)=q g+r .
$$

To prove that $\mathcal{A}_{X, x}$ is Noetherian is clearly suffices to show that:

For any $f \in \mathcal{A}_{X, x}$ the ring $\mathcal{A}_{X, x} / \mathcal{A}_{X, x} f$ is Noetherian .

We perform a change of coordinates as in (3.11) so that by (3.15) we can assume that $f$ is $t$-regular.

The Weierstrass preparation theorem follows from the Weierstrass division theorem formally and it implies, with our hypotheses on $f$, that we have

$$
f=u g \quad u \in \mathcal{A}_{X, x} \text { is a unit } g \in \mathcal{A}_{X^{\prime}, x}\left[x_{n}\right] .
$$

Therefore

$$
\mathcal{A}_{X, x} / \mathcal{A}_{X, x} f \cong \mathcal{A}_{X, x} / \mathcal{A}_{X, x} g \cong \mathcal{A}_{X^{\prime}, x}\left[x_{n}\right] / \mathcal{A}_{X^{\prime}, x}\left[x_{n}\right] g .
$$

But, by induction, we conclude that $\mathcal{A}_{X^{\prime}, x}\left[x_{n}\right]$ is Noetherian and hence so is $\mathcal{A}_{X, x} / \mathcal{A}_{X, x} f$. To see the second isomorphism we apply the division theorem again.

Proposition 3.8. Let $k<h$ then $\mathcal{A}_{X, x}^{\hat{k}}$ is faithfully flat over $\mathcal{A}_{X, x}^{\hat{h}}$.

Proof. We make use of the appendix of Se] where the notion of (faithful) flatness was originally introduced. The local ring is $\mathcal{A}_{X, x}^{\hat{h}}$ is a subring of $\mathcal{A}_{X, x}^{\hat{k}}$ and their formal completions coincide. Now we use the fact that the formal completion $\hat{B}$ of a regular local ring $B$ is faithfully flat over $B$. This implies that $\mathcal{A}_{X, x}^{\hat{k}}$ is faithfully flat over $\mathcal{A}_{X, x}^{\hat{h}}$.

Proposition 3.9. The sheaf $\mathcal{A}_{X}$ is coherent.

Proof. Note that $\mathcal{A}_{X}$ is naturally a filtered ring and the associated graded $\operatorname{gr} \mathcal{A}_{X} \cong$ $\mathcal{O}_{X}[t]$ is a coherent. Now, we just checked that $\mathcal{A}_{X, x}$ is Noetherian and it is Zariskian, because it is a local ring. Then $\mathcal{A}_{X}$ is coherent by [Sch, Chapter II, proposition 1.4.1].

\section{The CASE OF VARYING LEVELS}

In our proof of the Cartan theorems we have to allow the level $h$ to vary. We introduce this generality already here as the proofs are the same as in the constant case.

Given a continuous function $h: X \rightarrow \mathbb{R}_{+}$, we can consider the corresponding sheaf $\mathcal{A}_{X}^{h}$ on $X$ defined as follows:

(4.1) $\mathcal{A}_{X}^{h}(U)=\left\{f: U \rightarrow \hat{A}=\mathbb{C}[[t]] \mid f\right.$ holomorphic and $f(x) \in A_{h(x)}$ for all $\left.x \in U\right\}$. 
We also have the corresponding sheaf $\mathcal{A}_{X}^{\check{h}}$ obtained as a direct limit

$$
\mathcal{A}_{X}^{\check{h}}=\underset{h_{1}>h}{\underset{\lim }{\longrightarrow}} \mathcal{A}_{X}^{h_{1}}
$$

here $h_{1}>h$ means that $h_{1}(x)>h(x)$ for all $x$.

Let us consider the stalks of the sheaves $\mathcal{A}_{X}^{\breve{h}}$. We claim that

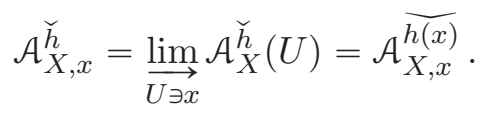

To see this, note that

For any $h_{1}>h$ there exists a neighborhood $U_{1}$ of $x$ such that $h_{1}(y)>h(x)$ for any $y \in U_{1}$.

Now, any $s \in \lim _{U \ni x} \mathcal{A}_{X}^{\breve{h}}(U)$ is given by $s \in \mathcal{A}_{X}^{\breve{h}}(U)$ for some $U \ni x$. Restricting $s$ further to a neighborhood $V \ni x$ with compact closure we conclude that there is an $h_{1}>h$ such that $s \in \mathcal{A}_{X}^{h_{1}}(V)$. If necessary we shrink $V$ further so that it is contained in $U_{1}$ given by (4.3). Thus, $s$ gives rise to an element in $\mathcal{A}_{X}^{h_{1}(x)}(V)$ and hence an element in $\mathcal{A}_{X, x}^{h(x)}$. This gives a map $\mathcal{A}_{X, x}^{\breve{h}} \rightarrow \mathcal{A}_{X, x}^{h(x)}$. It is now easy to see that this map is an isomorphism.

Remark 4.1. Note that we do not have an analogous statement for $\mathcal{A}_{X}^{h}$.

We have

Proposition 4.2. The sheaf $\mathcal{A}_{X}^{\check{h}}$ is coherent.

The proof of this proposition is the same as the proof of 3.9

Let us now consider an $\mathcal{A}_{X}^{\breve{h}}$-coherent sheaf $\mathcal{F}$. First, given any $x \in X$ and using the fact that the local ring $\mathcal{A}_{X, x}$ is regular we obtain a resolution $\mathcal{L}^{x}$ of $\mathcal{F}_{x}$ of length $n+1$ with the $\mathcal{L}_{k}^{x}$ free $\mathcal{A}_{X, x}$-modules of finite rank. Thus, there exists a neighborhood $V_{x}$ of $x$ such that the connecting homomorphisms $\partial_{i}: \mathcal{L}_{i}^{x} \rightarrow \mathcal{L}_{i-1}^{x}$ are defined on $V_{x}$ and so we can view $\mathcal{L}^{x}$. as a complex of free $\mathcal{A}_{V_{x}}$-modules of finite rank on $V_{x}$. The homology groups $H_{k}\left(\mathcal{L}^{x}\right)$ are coherent sheaves on $V_{x}$ such that the stalks $H_{k}\left(\mathcal{L}^{x}\right)_{x}=0$. Thus, possibly by shrinking $V_{x}$ the complex $\mathcal{L}^{x}$. is a resolution of $\left.\mathcal{F}\right|_{V_{x}}$.

In particular, given any $x \in X$ there is neighborhood $V_{x}$ of $x$ such that $\left.\mathcal{F}\right|_{V_{x}}$ has a free resolution $\mathcal{L}$.. We can shrink the $V_{x}$ so that the new $V_{x}$ has compact closure in the old one. This allows us to assume that the maps in the complex $\mathcal{L}$. are defined over $\mathcal{A}_{X}^{h^{\prime}}$ for an $h^{\prime}>h$. So, if $\mathcal{L}$. is given by

$$
\mathcal{L} .=\cdots \rightarrow\left(\mathcal{A}_{V_{x}}^{\check{h}}\right)^{\oplus p_{k}} \rightarrow \cdots \rightarrow\left(\mathcal{A}_{V_{x}}^{\check{h}}\right)^{\oplus p_{1}} \rightarrow\left(\mathcal{A}_{V_{x}}^{\check{h}}\right)^{\oplus p_{0}}
$$

then for any $k$ with $h^{\prime} \geqslant k>h$ we can form the complex $\mathcal{L}^{k}$.

$$
\mathcal{L}^{k}=\cdots \rightarrow\left(\mathcal{A}_{V_{x}}^{k}\right)^{\oplus p_{k}} \rightarrow \cdots \rightarrow\left(\mathcal{A}_{V_{x}}^{k}\right)^{\oplus p_{1}} \rightarrow\left(\mathcal{A}_{V_{x}}^{k}\right)^{\oplus p_{0}} ;
$$

note that $\mathcal{L}^{k}$ it is just a complex and we make no claims about vanishing of any of its cohomology groups. To make it a resolution we pass to the direct limit as follows. For 
any $h^{\prime}>h_{1}>h$ we then have

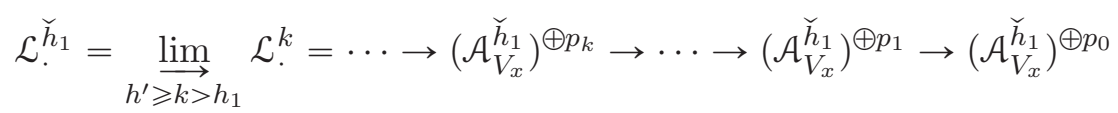

Now, clearly,

$$
\mathcal{L} .=\mathcal{A}_{V_{x}}^{\check{h}} \otimes_{\mathcal{A}_{V_{x}}^{\breve{h}_{1}}} \mathcal{L}^{\check{L}_{1}}
$$

Passing to the level of stalks we obtain

$$
(\mathcal{L} .)_{x}=\mathcal{A}_{X, x}^{\check{h}} \otimes_{\mathcal{A}_{X, x}^{\breve{h}_{1}}}\left(\mathcal{L}^{\breve{h}}\right)_{x} .
$$

As $\mathcal{A}_{X, x}^{\breve{h}}$ is faithfully flat over $\mathcal{A}_{X, x}^{\check{h}_{1}}$ we conclude that $H_{k}\left(\left(\mathcal{L}^{\breve{h}_{1}}\right)_{x}\right)=0$ for $k \geqslant 1$ because that is the case for $(\mathcal{L} .)_{x}$ and then, finally, that

$$
H_{k}\left(\check{L}^{\breve{h}_{1}}\right)=0 \quad \text { for } k \geqslant 1 .
$$

We now set

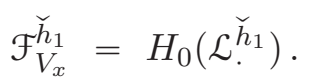

Note that, by construction, we also then have for $h_{1} \geqslant h_{2} \geqslant h$

$$
\mathcal{F}_{V_{x}}^{\check{h}_{2}} \cong \mathcal{A}_{V_{x}}^{\check{h}_{2}} \otimes_{\mathcal{A}_{V_{x}}^{\breve{h}_{1}}} \mathcal{F}_{V_{x}}^{\check{h}_{1}}
$$

In particular,

$$
\left.\mathcal{F}\right|_{V_{x}} \cong \mathcal{A}_{V_{x}} \otimes_{\mathcal{A}_{V_{x}}^{\breve{h}_{1}}} \mathscr{\mathcal { F }}_{V_{x}}^{\breve{h}_{1}}
$$

By faithful flatness we have a canonical inclusion

$$
\left.\mathcal{F}_{V_{x}}^{\check{h}_{1}} \subset \mathcal{F}\right|_{V_{x}} \text {. }
$$

The constructions that we have just performed depend on the choice of the initial resolution $\mathcal{L}$.. We will next study this dependence. We can do this in two ways. One is to work to with compact neighborhoods of $x$ to begin with or we work with the open neighborhoods $V_{x}$, but always think of them as being equipped with an open neighborhood $U_{x}$ of $x$ such that $U_{x} \subset \bar{U}_{x} \subset V_{x}$ with $\bar{U}_{x}$ compact. Let us choose a cover of $X$ by the $U_{x}$, with $x \in I$ such that the only finitely many $V_{x}$ have a non-empty intersection.

Now, for each $V_{x}$ we obtain a particular function $l_{x}$ as above. Furthermore, for each pair of $x, y$ such that $U_{x} \cap U_{y}$ is non-empty, we can compare the resolutions $\mathcal{L}$. $(x)$ and $\mathcal{L}$.(y) by first restricting them to $V_{x} \cap V_{y}$. So, we have on $V_{x} \cap V_{y}$

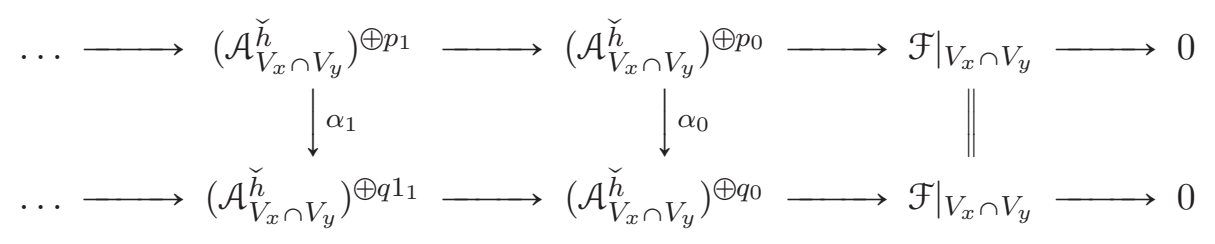


where the vertical arrows $\alpha_{i}$ are lifts of the identity map. Now the maps $\alpha_{i}$ and the maps to the opposite direction are defined on some particular level $l_{x, y}$ with $l_{x, y}>h$. We now find a $k_{x}$ on $U_{x}$ such that

$$
l_{x} \geqslant k_{x}>h \text { and } l_{x, y} \geqslant k_{x}, l_{y} \geqslant k_{x} \text { for all } y \text { such that } U_{x} \cap U_{y} \text { not empty . }
$$

For any $k$ such that $k_{x}>k>h$ we can now construct the sheaf $\mathcal{F}_{U_{x}}^{\breve{k}}$ by making use of the resolution $\mathcal{L}$. $(x)$. Furthermore, if $U_{x} \cap U_{y}$ in not empty then, by our choice of $D_{x}$ we get an isomorphism between $\left.\mathcal{F}_{U_{x}}^{\breve{k}}\right|_{U_{x} \cap U_{y}}$ and $\left.\mathcal{F}_{U_{y}}^{\breve{k}}\right|_{U_{x} \cap U_{y}}$. This isomorphism becomes, by construction, the identity when we extend scalars to $\mathcal{A}_{X}^{\check{h}}$. This allows us to identify $\left.\mathcal{F}_{U_{x}}^{\breve{k}}\right|_{U_{x} \cap U_{y}}$ and $\left.\mathcal{F}_{U_{y}}^{\breve{k}}\right|_{U_{x} \cap U_{y}}$ as sub sheaves of $\left.\mathcal{F}\right|_{U_{x} \cap U_{y}}$. So we can glue the sheaves to obtain a sheaf $\mathcal{F}_{U_{x}}^{\breve{k}} \cup U_{y}$.

Finally, we now choose a function $k$ such that $k>h$ and $k_{x} \geqslant k$ for all $x \in X$. Once we have done this then for any $h_{1}$ with $k>h_{1}>h$ we have constructed sheaves $\mathcal{F}^{\breve{h}_{1}}$ which coincide with the $\mathscr{F}_{U_{x}}^{\breve{h}_{1}}$ on $U_{x}$ and which thus satisfy

$$
\mathcal{F} \cong \mathcal{A}_{X}^{\check{h}} \otimes_{\mathcal{A}_{X}^{\breve{h}_{1}}} \mathcal{F}^{\breve{h}_{1}}
$$

Furthermore, we have a canonical inclusion:

$$
\mathscr{F}^{\check{h}_{1}} \subset \mathcal{F} \text {. }
$$

\section{CARTAN TheOREMS For COHEREnT $\mathcal{A}_{X}^{\check{h}}$-MODUles ON COMPACT BLOCKS}

In this section we prove Cartan's theorem for compact blocks in $\mathbb{C}^{N}$ for certain varying levels $h(x)$. We restrict our attention to blocks mainly for simplicity of exposition as this is enough for our arguments in the rest of the paper. We make use of the $L^{2}$-methods of Hörmander as they seem best suited to this task.

We will denote the coordinates in $\mathbb{C}^{N}$ by $z=\left(z_{1}, \ldots, z_{N}\right)$. Recall that compact blocks $Q \subset \mathbb{C}^{N}$ are products of rectangles $R=\{z \in \mathbb{C} \mid a \leqslant \operatorname{Re} z \leqslant b, c \leqslant \operatorname{Im} z \leqslant d\}$. We allow for the possibility of degenerate rectangles and argue by induction on the dimension of $Q$. We write $r=\sup \left\{\left|z_{1}\right|, \ldots,\left|z_{n}\right|\right\}$ and use it as a norm on $Q$.

We consider functions $h: Q \rightarrow \mathbb{R}^{+}$which only depend on $r$ and which are twice differentiable as functions of $r$ satisfying the following condition:

$$
h^{\prime}(r)^{2} \geqslant h(r) h^{\prime \prime}(r) \quad \text { for all } r>0 .
$$

Let us note that it is easy to characterize such functions. We write

$$
h(r)=\exp \left(-\int_{0}^{r} H(s) d s\right) .
$$

The condition (5.1) is then equivalent to

$$
H(r)^{2} \geqslant-H^{\prime}(r)+H(r)^{2} \text { for all } r>0 .
$$

This condition holds precisely as long as $H^{\prime}(r) \geqslant 0$, i.e., as long as $H(r)$ is nondecreasing. 
Theorem 5.1. If $h$ satisfies condition (5.1) and $\mathcal{F}$ is a coherent $\mathcal{A}_{X_{\nu}}^{\breve{h}}$-module defined on a neighborhood of $Q$ then $\mathrm{H}^{k}(Q, \mathcal{F})=0$ for $k \geqslant 1$ and $\mathcal{F}$ is generated by global sections in the strong sense, i.e., there is an $n$ and a surjection $\left(\mathcal{A}_{Q}^{\breve{h}}\right)^{\oplus n} \rightarrow \mathcal{F}$.

Remark 5.2. Note that we can view $\mathcal{A}_{Q}^{\check{h}}$ as specifying an analogue of a compact domain. According to the proposition when $h$ satisfies condition (5.1) this domain is holomorhically convex. We can view such domains as compact neighborhoods of $Q$ in $Q \times \mathbb{C}$. By the discussion above such holomorphically convex domains are plentiful and form a basis of all compact neighborhoods.

We prove the following theorem using Dolbeault cohomology and standard $L^{2}$ methods.

Proposition 5.3. If $Q$ is a compact block and $h$ satisfies condition (5.1) then $\mathrm{H}^{k}\left(Q, \mathcal{A}_{Q}^{\breve{h}}\right)=$ 0 for $k \geqslant 1$. Furthermore, if $E$ is a locally free $\mathcal{A}_{\mathbb{C}^{N}}^{\check{h}}$-module of finite rank (i.e., an $\mathcal{A}_{\mathbb{C}^{N}}^{\check{h}}$ vector bundle) defined in a neighborhood of $Q$ then $\mathrm{H}^{k}(Q, E)=0$ for $k \geqslant 1$.

First of all, we prove the theorem by proving that the (global) Dolbeault complex is exact. To explain the Dolbeault complex let us return temporarily to the case of an arbitrary complex manifold $X$. First of all let us consider the classical Dolbeault complex on $X$ :

$$
C_{X}^{\infty}=\Omega_{X}^{0,0} \stackrel{\bar{\partial}}{\rightarrow} \Omega_{X}^{0,1} \stackrel{\bar{\partial}}{\rightarrow} \ldots \stackrel{\bar{\partial}}{\rightarrow} \Omega_{X}^{0, q} \stackrel{\bar{\partial}}{\rightarrow}
$$

which gives us a resolution of $\mathcal{O}_{X}$. Let us write $\mathcal{C}_{X}^{\infty, \breve{h}}$ for the smooth version of $\mathcal{A}_{X}^{\breve{h}}$, i.e., we first set:

$$
\begin{gathered}
\mathcal{C}_{X}^{\infty, h}(U)=\left\{f: U \rightarrow \hat{A}=\mathbb{C}[[t]] \mid f \text { smooth and } \frac{\partial^{\alpha} f}{\partial x^{\alpha}}(x) \in A_{h(x)}\right. \\
\text { for all } x \in U \text { and all multi indices } \alpha\} .
\end{gathered}
$$

and then pass to a direct limit. Note that if $h(x)=k$ is constant then $\mathcal{C}_{X}^{\infty, k}(U)$ agrees with the usual notion of smooth functions with values in $A_{k}$ and then $\mathcal{C}_{X}^{\infty, k}(U)=$ $A_{h} \hat{\otimes}_{\mathbb{C}} C_{X}^{\infty}(U)$. This follows from the fact, which is easy to verify in our case directly that continuity and continuous differentiability can be checked component wise (weak continuity/differentiability).

We can now form the $\bar{\partial}$-complex of $\mathcal{A}_{X}^{\check{h}}$ by tensoring the above complex (5.4) with $\mathcal{C}_{X}^{\infty, \breve{h}}$ over $C_{X}^{\infty}$. Let us write

$$
\mathcal{A}_{X}^{\check{h},(0, q)}=\mathcal{C}_{X}^{\infty, \breve{h}} \otimes_{C_{X}^{\infty}} \Omega_{X}^{0, q}
$$

and then we obtain a complex

$$
\mathcal{C}_{X}^{\infty, \breve{h}}=\mathcal{A}_{X}^{\breve{h},(0,0)} \stackrel{\bar{\partial}}{\rightarrow} \mathcal{A}_{X}^{\breve{h},(0,1)} \stackrel{\bar{\partial}}{\rightarrow} \ldots \stackrel{\bar{\partial}}{\rightarrow} \mathcal{A}_{X}^{\breve{h},(0, q)} \stackrel{\bar{\partial}}{\rightarrow} .
$$

Finally if $E$ is locally free $\mathcal{A}_{X}^{\breve{h}}$ sheaf we write $\mathcal{E}=\mathcal{C}_{X}^{\infty, \breve{h}} \otimes_{\mathcal{A}_{X}^{\check{h}}} E$ for the smooth version and we also write

$$
\mathcal{E}_{X}^{\breve{h},(0, q)}=\mathcal{E} \otimes_{\mathcal{C}_{X}^{\infty, \breve{h}}} \mathcal{A}_{X}^{\check{h},(0, q)}
$$


and we obtain a complex

$$
\mathcal{E}=\varepsilon_{X}^{\check{h},(0,0)} \stackrel{\bar{\partial}}{\rightarrow} \varepsilon_{X}^{\breve{h},(0,1)} \stackrel{\bar{\partial}}{\rightarrow} \ldots \stackrel{\bar{\partial}}{\rightarrow} \varepsilon_{X}^{\check{h},(0, q)} \stackrel{\bar{\partial}}{\rightarrow} ;
$$

the operator $\bar{\partial}$ is well-defined because $E$ is a holomorphic $\mathcal{A}_{X}^{\breve{h}}$-bundle. The complexes (5.5) and (5.6) are resolutions of $\mathcal{A}_{X}^{\breve{h}}$ and $E$, respectively. One can deduce this from the exactness of (5.4) as follows. As exactness is a local question, the exactness of (5.6) follows from that of (5.5). We have, passing to stalks:

$$
\mathcal{A}_{X, x}^{\breve{h},(0, q)}=\mathcal{C}_{X, x}^{\infty, \breve{h}} \otimes_{C_{X, x}^{\infty}} \Omega_{X, x}^{0, q} .
$$

Now, arguing just as for (4.3) in case of $\mathcal{A}_{X, x}^{\breve{h}}$, we have

$$
\mathcal{C}_{X, x}^{\infty, \breve{h}}=\mathcal{C}_{X, x}^{\overline{h(x)}}
$$

and, finally, by $[\mathrm{Ko}, \S 41(6) \mathrm{b})]$,

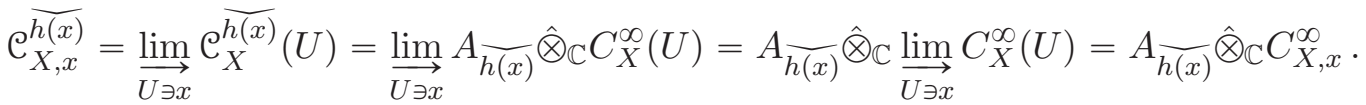

Putting things together, we conclude that

$$
\mathcal{A}_{X, x}^{\breve{h},(0, q)}=A_{\overline{h(x)}} \hat{\otimes}_{\mathbb{C}} C_{X, x}^{\infty} \otimes_{C_{X, x}^{\infty}} \Omega_{X, x}^{0, q}=A_{\overline{h(x)}} \hat{\otimes}_{\mathbb{C}} \Omega_{X, x}^{0, q} .
$$

The exactness of (5.5), and hence that of (5.6), follow from the exactness of (5.4) because $A_{h(x)} \hat{\otimes}_{\mathbb{C}}$ is an exact functor on DF-spaces by Lemma 2.4.

Finally, as $\mathcal{A}_{X}^{\breve{h},(0, q)}$ and $\mathcal{E}_{X}^{\breve{h},(0, q)}$ are modules over $C_{X}^{\infty}$ they are soft and hence acyclic. Thus we have reduced the proof of Proposition 5.3 to the following:

Proposition 5.4. If $Q$ is a compact block in $\mathbb{C}^{N}$ and $h$ satisfies condition (5.1) then the complex

$$
\mathcal{A}_{\mathbb{C}^{N}}^{\check{h},(0,0)}(Q) \stackrel{\bar{\partial}}{\rightarrow} \mathcal{A}_{\mathbb{C}^{N}}^{\breve{h},(0,1)}(Q) \stackrel{\bar{\partial}}{\rightarrow} \ldots \stackrel{\bar{\partial}}{\rightarrow} \mathcal{A}_{\mathbb{C}^{N}}^{\check{h},(0, q)}(Q) \stackrel{\bar{\partial}}{\rightarrow}
$$

is exact in degrees $q \geqslant 1$. Similarly, if $E$ is a locally free $\mathcal{A}_{\mathbb{C}^{N}}^{\breve{h}}$-module of finite rank (i.e., an $\mathcal{A}_{\mathbb{C}^{N}}^{\check{h}}$ vector bundle) defined in a neighborhood of $Q$ then the complex

$$
\varepsilon_{\mathbb{C}^{N}}^{\check{h},(0,0)}(Q) \stackrel{\bar{\partial}}{\rightarrow} \varepsilon_{\mathbb{C}^{N}}^{\check{h},(0,1)}(Q) \stackrel{\bar{\partial}}{\rightarrow} \ldots \stackrel{\bar{\partial}}{\rightarrow} \varepsilon_{\mathbb{C}^{N}}^{\check{h},(0, q)}(Q) \stackrel{\bar{\partial}}{\rightarrow}
$$

is exact in degrees $q \geqslant 1$.

We postpone the proof of this proposition to the next section. However, let us record the following lemma which explains the meaning of condition (5.1) as well as our initial requirement (3.4e). These conditions are chosen precisely so that the following lemma holds. Without plurisubharmonicity vanishing of cohomology would not hold in general.

Lemma 5.5. If h satisfies condition (5.1) then the function

$$
W_{j}: z \mapsto-2 \log \left\|t^{j}\right\|_{h(r)}, \quad z \in Q,
$$

is plurisubharmonic for every $j$. 
Proof. Recall the notation from (3.1). We first remark that (3.4e) implies, by the positivity of $N_{j}$ and a direct calculation of the derivatives, that

$$
\frac{\left(N_{j}^{\prime}(h)\right)^{2}}{N_{j}(h)}-N_{j}^{\prime \prime}(h)-\frac{1}{h} N_{j}^{\prime}(h) \geqslant 0
$$

We write $T_{j}:=N_{j} \circ h$; then

$$
W_{j}(z)=-2 \log T_{j}(r)
$$

and the plurisubharmonicity (5.8) follows, if we show that $-\frac{d^{2}}{d r^{2}} \log T_{j}(r) \geqslant 0$, for all $r \in \mathbb{R}^{+}, j \in \mathbb{N}$. (The composite of a convex, increasing function and a plurisubharmonic function is plurisubharmonic, see, for example, [Kr, Prop. 2.2.6.]) We have by (5.9)

$$
\begin{aligned}
& -\frac{d^{2} \log T_{j}}{d r^{2}}=\frac{\left(T_{j}^{\prime}\right)^{2}}{\left(T_{j}\right)^{2}}-\frac{T_{j}^{\prime \prime}}{T_{j}} \\
= & \frac{1}{N_{j}}\left(\frac{\left(N_{j}^{\prime}\right)^{2}\left(h^{\prime}\right)^{2}}{N_{j}}-\left(N_{j}^{\prime \prime}\right)\left(h^{\prime}\right)^{2}-\left(N_{j}^{\prime}\right) h^{\prime \prime}\right) \\
= & \frac{1}{N_{j}}\left(\left(h^{\prime}\right)^{2}\left(\frac{\left(N_{j}^{\prime}\right)^{2}}{N_{j}}-N_{j}^{\prime \prime}\right)-\left(N_{j}^{\prime}\right) h^{\prime \prime}\right) \\
\geqslant & \frac{1}{N_{j}}\left(\frac{\left(h^{\prime}\right)^{2}}{h}-h^{\prime \prime}\right) N_{j}^{\prime}
\end{aligned}
$$

Since $N_{j}$ is always positive and increasing, the required positivity of follows from (5.1).

We will now deduce Theorem 5.1 from Proposition 5.3. We will proceed in complete analogy with $[\mathrm{H}$, Section 7.2] with the simplification that we work with compact blocks. As was mentioned before, this assumption is not necessary but it suffices for us. The argument in Hörmander can be repeated word for word. Thus, we explain the argument only briefly. We will argue by induction on the dimension of $Q$. In the case when the dimension of $Q$ is zero there is nothing to prove. Let us consider $Q$ of a particular dimension $d$ and let us fiber $Q$ as follows

$$
\pi: Q \rightarrow[a, b]
$$

The fibers of $\mathrm{Q}$ are now compact blocks of dimension $d-1$. Each fiber $\pi^{-1}(e)$ thus satisfies Cartan A. Let us now fix a coherent sheaf $\mathcal{F}$ in the neighborhood of $Q$. Thus, there is an open neighborhood $U_{e}$ of $\pi^{-1}(e)$ and a surjection $\left.\left(\mathcal{A}_{U_{e}}^{\breve{h}}\right) \oplus p_{e} \rightarrow \mathcal{F}\right|_{U_{e}}$. We can choose the neighborhood $U_{e}$ to be rectangle $Q_{e} \times\left[a_{e}, b_{e}\right]$. We now choose a finite sub cover $\left\{U_{i}\right\}_{i \in I}$ of $U_{e}$ shrinking the $U_{e}$, if necessary, so that the $U_{i}$ have the form

$$
U_{i}=\tilde{Q} \times\left(a_{i}, b_{i}\right) \text { with } a_{1}<a_{2}<b_{1}<a_{3}<b_{2}<\ldots a_{n}<b_{n-1}<b_{n} .
$$


In other words only the consecutive $U_{i}$ intersect each other. On each overlap $U_{i} \cap U_{i+1}$ we choose $\alpha_{i}$ and $\beta_{i}$ making the diagram below commute:

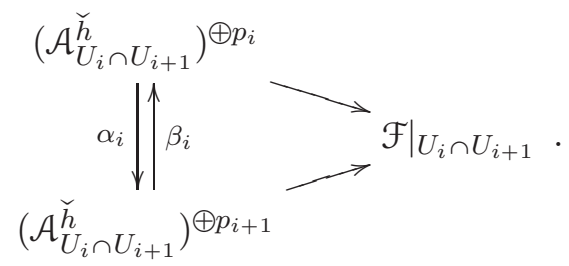

We now use the $\alpha_{i}$ and $\beta_{i}$ to construct transition functions, as in $[\underline{\mathrm{H}}$, Section 7.2]. As there are no triple intersections of the $U_{i}$ the transition functions yield a vector bundle $E$ on $U=\cup U_{i}$ and a surjection $\left.E \rightarrow \mathcal{F}\right|_{U}$. By writing $\mathcal{K}_{1}$ for the kernel of the map $\left.E \rightarrow \mathcal{F}\right|_{U}$ we obtain an exact sequence

$$
0 \rightarrow \mathcal{K}_{1} \rightarrow\left(\mathcal{A}_{U}^{\check{h}}\right)^{p} \rightarrow \mathcal{F} \rightarrow 0
$$

Thus, making use of proposition 5.3 for $i \geqslant 1$ we get that

$$
\mathrm{H}^{i}(Q, \mathcal{F}) \cong \mathrm{H}^{i+1}\left(Q, \mathcal{K}_{1}\right)
$$

Repeating this argument for the coherent sheaf $\mathcal{K}_{j}$ and always writing $\mathcal{K}_{j+1}$ for the kernel we get:

$$
\mathrm{H}^{i}(Q, \mathcal{F}) \cong \mathrm{H}^{i+1}\left(Q, \mathcal{K}_{1}\right) \cong \cdots \cong \mathrm{H}^{i+p}\left(Q, \mathcal{K}_{p}\right)
$$

When $p \geqslant 2 N$ the right hand side vanishes and Cartan B follows for $Q$. It remains to prove Cartan A for $Q$.

Let $x \in Q$ and write $i_{x}:\{x\} \rightarrow Q$. Then we have the following exact sequence

$$
0 \rightarrow \mathcal{K}_{x} \rightarrow \mathcal{F} \rightarrow\left(i_{x}\right)_{*}\left(i_{x}\right)^{*} \mathcal{F} \rightarrow 0
$$

Taking the long exact sequence and using Cartan B we obtain a surjection

$$
\mathcal{F}(Q) \rightarrow\left(i_{x}\right)_{*}\left(i_{x}\right)^{*} \mathcal{F}=\mathcal{F}_{x} / \mathfrak{m}_{x} \mathcal{F}_{x},
$$

where $\mathfrak{m}_{x}$ is the maximal ideal in $\mathcal{A}_{X, x}^{\breve{h}}=\mathcal{A}_{X, x}^{\overline{h(x)}}$. As $\left(i_{x}\right)_{*}\left(i_{x}\right)^{*} \mathcal{F}=\mathcal{F}_{x} / \mathfrak{m}_{x} \mathcal{F}_{x}$ is coherent it is finitely generated over $A_{h(x)}$. This implies that there are finitely many sections of $\mathcal{F}(Q)$ which $\operatorname{span}\left(i_{x}\right)_{*}\left(i_{x}\right) * \mathcal{F}=\mathcal{F}_{x} / \mathfrak{m}_{x} \mathcal{F}_{x}$. By Nakayama's lemma these sections also span $\mathcal{F}_{x}$ over $\mathcal{A}_{X, x}^{\breve{h}}$. Thus we obtain a map

$$
\left(\mathcal{A}_{Q}^{\check{h}}\right)^{\oplus n_{x}} \rightarrow \mathcal{F}
$$

which is a surjection on the level of stalks at $x$. Thus, it is a surjection on some open neighborhood $U_{x}$ of $x$. As $Q$ is compact, it can be covered by a finite number of the $U_{x}$, say, $U_{x_{1}}, \ldots, U_{x_{s}}$. Then

$$
\oplus_{i=1}^{s}\left(\mathcal{A}_{Q}^{\breve{h}}\right)^{\oplus n_{x_{i}}} \rightarrow \mathcal{F}
$$

is the required surjection. 


\section{Proof of Proposition 5.4}

We retain the notation of the previous section. In particular, we denote the coordinates in $\mathbb{C}^{N}$ by $z=\left(z_{1}, \ldots, z_{N}\right)$ and the Lebesgue measure on $\mathbb{C}^{N}$ by $d x$. As before, $Q$ stands for a compact block.

6.1. The structure sheaf case. We begin by construction the $L^{2}$-version of the $\bar{\partial}$ complex

$$
\mathcal{A}_{Q}^{\breve{h},(0,0)}(Q) \stackrel{\bar{\partial}}{\rightarrow} \mathcal{A}_{Q}^{\breve{h},(0,1)}(Q) \stackrel{\bar{\partial}}{\rightarrow} \ldots \stackrel{\bar{\partial}}{\rightarrow} \mathcal{A}_{Q}^{\check{h},(0, q)}(Q) \stackrel{\bar{\partial}}{\rightarrow}
$$

in complete analogy with the classical $L^{2}$-version of the $\bar{\partial}$-complex. We could as easily work with $(p, q)$-forms, but in what follows we will stick to $(0, q)$-forms to simplify the notation. We will construct a complex, but for an $h(r)$, not $\overline{h(r)}$, of the following form:

$$
L_{(0,0)}^{2}\left(Q ; H_{h(r)}\right) \stackrel{\bar{\partial}}{\rightarrow} L_{(0,1)}^{2}\left(Q ; H_{h(r)}\right) \stackrel{\bar{\partial}}{\rightarrow} \ldots \stackrel{\bar{\partial}}{\rightarrow} L_{(0, q)}^{2}\left(Q ; H_{h(r)}\right) \stackrel{\bar{\partial}}{\rightarrow} .
$$

The space $L_{(0, q)}^{2}\left(Q ; H_{h(r)}\right)$ is an $L^{2}$-spaces of forms with values in a varying Hilbert spaces $H_{h(r)}$; we will soon define these and also give the precise meaning of the vector valued $\bar{\partial}$ operator.

Let us recall the algebra norms (3.7), $\left\|\sum_{j} a_{j} t^{j}\right\|_{h(r)}=\sum_{j}\left|a_{j}\right|\left\|t^{j}\right\|_{h(r)}$ on the spaces $A_{h(r)}$. We introduce $L^{2}$-version of the spaces $A_{h(r)}$ and of the norms as follows:

$$
\begin{gathered}
H_{h(r)}=\left\{a=\sum_{j} a_{j} t^{j}:\|a\|_{2, h(r)}^{2}<\infty\right\}, \quad \text { where } \\
\|a\|_{2, h(r)}^{2}=\sum_{j=0}^{\infty}\left|a_{j}\right|^{2}\left\|t^{j}\right\|_{h(r)}^{2} .
\end{gathered}
$$

We define the space $L^{2}\left(Q ; H_{h(r)}\right)=L_{(0,0)}^{2}\left(Q ; H_{h(r)}\right)$ as a completion of the space of smooth functions $\mathcal{C}_{Q}^{\infty, h}$ (note that there is no hat in this formula). First, we set

$$
\left\|f ; L^{2}\left(Q ; H_{h(r)}\right)\right\|^{2}=\int_{Q}\|f(x)\|_{2, h(r)}^{2} d x
$$

for $\mathcal{C}_{Q}^{\infty, h}$ and then we complete it to $L^{2}\left(Q ; H_{h(r)}\right)$ with respect to the norm above. As we are working on $\mathbb{C}^{N}$, the bundles of forms $\Omega^{0, q}$ are naturally trivialized by choosing the $d \bar{z}^{\alpha}$ as a basis. Thus, we can define a norm on $\omega \in \mathcal{A}_{Q}^{h,(0, q)}(Q)$ by the formula

$$
\left\|\omega ; L_{0, q}^{2}\left(Q ; H_{h(r)}\right)\right\|^{2}=\sum_{\alpha}\left\|f_{\alpha} ; L^{2}\left(Q ; H_{h(r)}\right)\right\|^{2}
$$

for $\omega=\sum_{|\alpha|=q} f_{\alpha} d \bar{z}^{\alpha}$. We complete $\mathcal{A}_{Q}^{h,(0, q)}(Q)$ into the corresponding $L^{2}$-space $L_{(0, q)}^{2}\left(Q ; H_{h(r)}\right)$ with respect to the norm above.

To define the $\bar{\partial}$-operator let us rephrase our discussion in the language of $[\mathbf{H}$, Chapter 4]. We write $\omega=\sum \omega_{j} t^{j}$ and consider a particular $j$. Recall that by Lemma 5.5 $W_{j}(z)=-2 \log \left\|t^{j}\right\|_{h(r)}$ is plurisubharmonic and we can consider $W_{j}(z)$ as a plurisubharmonic weight. As in $\left[\underline{\mathrm{H}}\right.$, we write $L_{(0, q)}^{2}\left(Q, W_{j}\right)$ for forms which are square integrable 
with respect to the measure $e^{-W_{j}(z)} d x$. Then, by [ $\left.\mathrm{H}\right]$, the operator $\bar{\partial}$ is a closed, densely defined operator on the weighted $L^{2}$-space $L_{(0, q)}^{2}\left(Q, W_{j}\right)$ where differentiation is understood in the generalized sense. Denoting by $\mathcal{D}_{j} \subset L_{(0, q)}^{2}\left(Q, W_{j}\right)$ the domain of $\bar{\partial}$, we define the vector valued $\bar{\partial}$-operator in the space $L_{(0, q)}^{2}\left(Q ; H_{h(r)}\right)$ coordinate wise by setting its domain to be

$$
D(\bar{\partial})=\left\{u=\sum_{j} u_{j} t^{j}: u_{j} \in \mathcal{D}_{j}, \sum_{j}\left\|u_{j} ; L_{(0, q)}^{2}\left(Q, W_{j}\right)\right\|^{2}<\infty\right\} .
$$

It is straightforward to see that $\bar{\partial}$ becomes a densely defined and closed operator. These definitions give us the complex (6.2).

Lemma 6.1. Let $\omega \in L_{(0, q+1)}^{2}\left(Q ; H_{h(r)}\right)$ with $\bar{\partial} \omega=0$. Then the problem $\bar{\partial} u=\omega$ has $a$ solution such that $u$ belongs to $L_{(0, q)}^{2}\left(Q ; H_{h(r)}\right)$, and we have

$$
\left\|u ; L_{(0, q)}^{2}\left(Q ; H_{h(r)}\right)\right\| \leqslant c\left\|\omega ; L_{(0, q+1)}^{2}\left(Q ; H_{h(r)}\right)\right\| .
$$

Proof. We write

$$
\omega=\sum_{j} \omega_{j} t^{j}
$$

where the $\omega_{j}$ are now usual $L^{2}$-forms of type $(0, q)$ on $Q$. We have $\bar{\partial} \omega_{j}=0$ for every $j$. We now solve this $\bar{\partial}$-problem in a controlled way. Recall (5.8) where we defined the plurisubharmonic functions $W_{j}(z)=-2 \log \left\|t^{j}\right\|_{h(r)}$. Theorem 4.4.2 of [H] implies that for every $j$ the equation

$$
\bar{\partial} u_{j}=\omega_{j}
$$

has a solution $u_{j}=\sum_{\alpha} u_{\alpha, j}$ with a very specific bound:

$$
\sum_{\alpha} \int_{Q}\left|u_{\alpha, j}\right|^{2} e^{-W_{j}(z)}\left(1+r^{2}\right)^{-2} d x \leqslant \sum_{\alpha} \int_{Q}\left|f_{\alpha, j}\right|^{2} e^{-W_{j}(z)} d x
$$

here we have written $\omega_{j}=\sum_{\alpha} f_{\alpha, j}$.

Since the $Q$ is a compact block, the weight $\left(1+r^{2}\right)^{-2}$ is bounded from below by a constant $c^{-1}>0$ independent of $j$, and (6.7) implies

$$
\begin{gathered}
\sum_{\alpha} \int_{Q}\left|u_{\alpha, j}\right|^{2} e^{-W_{j}(z)} d x \leqslant c \sum_{\alpha} \int_{Q}\left|u_{\alpha, j}\right|^{2} e^{-W_{j}(z)}\left(1+r^{2}\right)^{-2} d x \leqslant \\
c \sum_{\alpha} \int_{Q}\left|f_{\alpha, j}\right|^{2} e^{-W_{j}(z)} d x
\end{gathered}
$$

We set

$$
u=\sum_{j} u_{j} t^{j}
$$


By (6.8) and the definitions of the norms in (6.3) and (6.4),

$$
\begin{aligned}
& \left\|u ; L_{(0, q)}^{2}\left(Q ; H_{h(r)}\right)\right\|^{2}=\sum_{j} \sum_{\alpha} \int_{Q}\left|u_{\alpha, j}\right|^{2}\left\|t^{j}\right\|_{h(r)}^{2} d x \\
= & \sum_{j} \sum_{\alpha} \int_{Q}\left|u_{\alpha, j}\right|^{2} e^{-W_{j}(z)} d x \leqslant c \sum_{j} \sum_{\alpha} \int_{Q}\left|f_{\alpha, j}\right|^{2} e^{-W_{j}(z)} d x \\
= & c \sum_{j} \sum_{\alpha} \int_{Q}\left|f_{\alpha, j}\right|^{2}\left\|t^{j}\right\|_{h(r)}^{2} d x=c\left\|f ; L_{(0, q+1)}\left(Q ; H_{h(r)}\right)\right\| .
\end{aligned}
$$

Thus we have constructed a solution to the $\bar{\partial}$-problem.

We have now proved the exactness of (6.2). We will use it to prove the required exactness of (6.1)

Let $\omega \in \mathcal{A}_{Q}^{\breve{h},(0, q)}(Q)$ such that $\bar{\partial} \omega=0$. We write, as usual, $\omega=\sum_{|\alpha|=q} f_{\alpha} d \bar{z}^{\alpha}$. Because of compactness of $Q$, we have

$$
\mathcal{A}_{Q}^{\breve{h}(r)}(Q)=\underset{m \rightarrow \infty}{\lim _{m}} \mathcal{A}_{Q}^{(1+1 / m) h(r)}(Q) .
$$

By compactness of $Q$ and continuity of $\omega$, there exist an $m$ such that $f_{\alpha}(z) \in A_{(1+1 / m) h(r)}$ for all $\alpha$ and all $z \in Q$. Moreover, obviously, for any $\left(a_{j}\right)_{j \in \mathbb{N}}, k$,

$$
\sum_{j}\left|a_{j}\right|\left\|t^{j}\right\|_{k} \geqslant\left(\sum_{j}\left|a_{j}\right|^{2}\left\|t^{j}\right\|_{k}^{2}\right)^{1 / 2} .
$$

Thus, we have the continuous embedding $A_{(1+1 / m) h(r)} \hookrightarrow H_{(1+1 / m) h(r)}$

Therefore every $f_{\alpha}$ is a continuous and thus bounded function $Q \rightarrow H_{(1+1 / m) h(r)}$, in particular, an element of $L^{2}\left(Q ; H_{(1+1 / m) h(r)}\right)$. Thus, $\omega \in L_{(0, q+1)}^{2}\left(Q ; H_{(1+1 / m) h(r)}\right)$ and by Lemma 6.1 we can find an $u \in L_{(0, q)}^{2}\left(Q ; H_{(1+1 / m) h(r)}\right)$ with $\bar{\partial} u=\omega$.

Writing $u=\sum u_{j} t^{j}$ and observing that $\bar{\partial}$-equation is an elliptic PDE with constant coefficients we conclude that the component functions $u_{j}$ are smooth functions.

We have an embedding

$$
H_{(1+1 / m) h(r)} \hookrightarrow A_{(1+1 /(m+1)) h(r)} .
$$

To see this we make use of our controlled nuclearity assumption (3.4d). It implies, in particular, that there is a constant $K$ such that

$$
\left\|t^{j}\right\|_{(1+1 /(m+1)) h(r)} \leqslant K j^{-1}\left\|t^{j}\right\|_{(1+1 / m) h(r)} \quad \text { for all } j .
$$

Making use of this inequality and the Cauchy-Schwartz inequality we conclude that

$$
\begin{aligned}
& \left\|\sum_{j} a_{j} t^{j}\right\|_{(1+1 /(m+1)) h(r)}=\sum_{j}\left|a_{j}\right|\left\|t^{j}\right\|_{(1+1 /(m+1)) h(r)} \leqslant \sum_{j}\left|a_{j}\right| K j^{-1}\left\|t^{j}\right\|_{(1+1 / m) h(r)} \\
\leqslant & K\left(\sum_{j}\left|a_{j}\right|^{2}\left\|t^{j}\right\|_{(1+1 / m) h(r)}^{2}\right)^{1 / 2} .
\end{aligned}
$$


This inequality gives us the embedding (6.10). Thus, we see that $u(z) \in A_{h_{1}(r)}$ for all $z \in Q$ with $h_{1}=(1+1 /(m+1)) h$. Finally, the operators $\frac{\partial^{\alpha}}{\partial x^{\alpha}}$ commute with the operator $\bar{\partial}$. Thus, $\bar{\partial} \frac{\partial^{\alpha} u}{\partial x^{\alpha}}=\frac{\partial^{\alpha} \omega}{\partial x^{\alpha}}$ and so we conclude that $\frac{\partial^{\alpha} u}{\partial x^{\alpha}}(z) \in A_{h_{1}(r)}$ for all $z \in Q$. Thus, we see that $u \in \mathcal{A}_{Q}^{\breve{h},(0, q)}(Q)$ proving the exactness of (6.1) in degrees $q \geqslant 1$.

6.2. The vector bundle case. Let us now turn to the case of a vector bundle $\mathrm{E}$ and write $n$ for its rank. The argument in this case will be bit more involved than in the case of the structure sheaf $\mathcal{A}_{Q}^{\breve{h}}$. In our case it is easy to write down an inner product

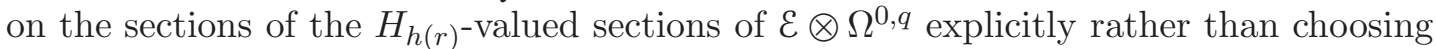
a Hermitian metric, so we proceed in this manner. This also makes it easier to reduce arguments to component functions as we did in the first part of this section.

Recall our $\bar{\partial}$-complex

$$
\varepsilon_{Q}^{\breve{h},(0,0)}(Q) \stackrel{\bar{\partial}}{\rightarrow} \varepsilon_{Q}^{\breve{h},(0,1)}(Q) \stackrel{\bar{\partial}}{\rightarrow} \ldots \stackrel{\bar{\partial}}{\rightarrow} \varepsilon_{Q}^{\breve{h},(0, q)}(Q) \stackrel{\bar{\partial}}{\rightarrow} .
$$

Just as in the case of $\mathcal{A}_{Q}^{\check{h}(r)}$ we will construct an analogous $L^{2}$-complex

$$
L_{(0,0)}^{2}\left(Q, \mathcal{E} ; H_{h(r)}\right) \stackrel{\bar{\partial}}{\rightarrow} L_{(0,1)}^{2}\left(Q, \mathcal{E} ; H_{h(r)}\right) \stackrel{\bar{\partial}}{\rightarrow} \ldots \stackrel{\bar{\partial}}{\rightarrow} L_{(0, q)}^{2}\left(Q, \mathcal{\varepsilon} ; H_{h(r)}\right) \stackrel{\bar{\partial}}{\rightarrow} .
$$

We will first prove that the $L^{2}$-complex is exact and then deduce from that, just as before, the exactness of (6.11).

The bundle $E$ is defined in an open neighborhood of $Q$. We choose finite open cover $Q_{i}^{\prime}$ of $Q$ by open blocks such that $\left.E\right|_{Q_{i}^{\prime}}$ is trivial. We now choose compact blocks $Q_{i} \subset Q_{i}^{\prime}$ such that the interiors $\AA_{i}$ also form an open cover of $Q$. We write $G_{i j}^{\prime}: Q_{i}^{\prime} \cap Q_{j}^{\prime} \rightarrow$ $G L_{n}\left(\mathcal{A}_{\mathbb{C}^{N}}^{\check{h}}\left(Q_{i}^{\prime} \cap Q_{j}^{\prime}\right)\right)$ for the transition matrices with respect to the cover $Q_{i}^{\prime}$. Let us write $G_{i j}=G_{i j}^{\prime} \mid Q_{i} \cap Q_{j}$. As the $Q_{i} \cap Q_{j}$ are compact there is an $h_{1}>h$ such that $G_{i j}: Q_{i} \cap Q_{j} \rightarrow G L_{n}\left(\mathcal{A}_{\mathbb{C}^{N}}^{h_{1}}\left(Q_{i} \cap Q_{j}\right)\right)$, for all $i, j$. Conversely, these transition functions give rise to vector bundle $E_{1}$ such that $E=\mathcal{A}_{\mathbb{C}^{N}}^{\breve{h}} \otimes_{\mathcal{A}_{\mathbb{C}^{N}}^{h_{1}}} E_{1}$ on some neighborhood of $Q$. For example, we can choose $h_{1}=(1+1 / m) h$ and then, of course, $h_{1}$ also satisfies condition (5.1). We will now work with $E_{1}$ and the function $h_{1}$.

We can view $L^{2}$ sections of $\mathcal{E}_{1}$ as a system of $n$-tuples $u_{i}=\left(u_{i, k}\right)_{k=1}^{n}, i=1, \ldots, I$, of functions $u_{i, k} \in L^{2}\left(Q_{i} ; H_{h_{1}(r)}\right)$ which satisfy

$$
u_{i}=G_{i j} u_{j} \quad \text { on } Q_{i} \cap Q_{j} .
$$

As before we trivialize the bundles of forms by our choice of coordinates on $\mathbb{C}^{N}$. Thus we can think of smooth $(0, q)$-forms of $\mathcal{E}_{1}$ as systems of $n$-tuples $u_{i}=\left(u_{i, k}\right)_{k=1}^{n}$ of $(0, q)$-forms $u_{i k} \in C_{(0, q)}^{\infty}\left(Q_{i} ; H_{h_{1}(r)}\right)$, with

$$
u_{i k}=\sum_{|\alpha|=q} u_{i, k, \alpha} d \bar{z}^{\alpha}
$$

where $u_{i, k, \alpha}$ is a $C^{\infty}$-function with values in $H_{h(r)}$ and where the $u_{i}$ satisfy (6.13). 
To define the space $L_{(0, q)}^{2}\left(Q, \mathcal{E}_{1} ; H_{h_{1}(r)}\right)$ of $L^{2}$-forms we consider the Hilbert space

$$
X(q)=\prod_{i=1}^{I} \prod_{k=1}^{n} L_{(0, q)}^{2}\left(Q_{i} ; H_{h_{1}(r)}\right),
$$

We then set

$$
\begin{gathered}
L_{(0, q)}^{2}\left(Q, \mathcal{E}_{1} ; H_{h_{1}(r)}\right)=\mathcal{H}(q)= \\
\left\{u=\left(u_{i}\right)_{i}=\left(u_{i k}\right)_{i, k} \in X(q) \mid u_{i}=G_{i j} u_{j} \text { for all } i, j\right\} .
\end{gathered}
$$

The norm $\|u\|_{q}$ on the space $\mathcal{X}(q)$ is associated to the natural inner product

$$
(g \mid v)_{q}=\sum_{i}\left(g_{i} \mid v_{i}\right)_{q, i}
$$

where $g=\left(g_{i k}\right)_{i k}, v=\left(v_{i k}\right)_{i k}$, and $(\cdot \mid \cdot)_{q, i}$ is the inner product of the Hilbert space $\prod_{k=1}^{n} L_{(0, q)}^{2}\left(Q_{i} ; H_{h_{1}(r)}\right)$.

We define the $\bar{\partial}$-operator on $\mathcal{H}(q)=L_{(0, q)}^{2}\left(Q, \mathcal{E}_{1} ; H_{h_{1}(r)}\right)$ as a restriction of the $\bar{\partial}$ operator on $X(q)$ which we define component wise. Since the coefficients $G_{i j}$ are analytic, we have from (6.13)

$$
\bar{\partial} u_{i}=G_{i j} \bar{\partial} u_{j}
$$

and hence we obtain $\bar{\partial}: L_{(0, q)}^{2}\left(Q, \mathcal{E}_{1} ; H_{h_{1}(r)}\right) \rightarrow L_{(0, q+1)}^{2}\left(Q, \mathcal{E}_{1} ; H_{h_{1}(r)}\right)$ and thus our complex

$$
L_{(0,0)}^{2}\left(Q, \mathcal{E}_{1} ; H_{h_{1}(r)}\right) \stackrel{\bar{\partial}}{\rightarrow} L_{(0,1)}^{2}\left(Q, \mathcal{E}_{1} ; H_{h_{1}(r)}\right) \stackrel{\bar{\partial}}{\rightarrow} \ldots \stackrel{\bar{\partial}}{\rightarrow} L_{(0, q)}^{2}\left(Q, \mathcal{E}_{1} ; H_{h_{1}(r)}\right) \stackrel{\bar{\partial}}{\rightarrow} .
$$

We will next prove the exactness of this complex, i.e.,

Proposition 6.2. Let $\omega \in L_{(0, q+1)}^{2}\left(Q, \varepsilon_{1} ; H_{h_{1}(r)}\right)$ with $\bar{\partial} \omega=0$. Then there exists $u \in L_{(0, q)}^{2}\left(Q, \mathcal{E}_{1} ; H_{h_{1}(r)}\right)$ such that $\bar{\partial} u=\omega$.

Let us pause to argue that this proposition implies the vector bundle part of Proposition 5.4. Let $\omega \in \mathcal{E}_{Q}^{\breve{h},(0, q+1)}(Q)$ such that $\bar{\partial} \omega=0$. By compactness of $Q$ there exists an $m$ such that $\omega$ is a smooth $(0, q)$ form of $\mathcal{E}_{1}$ with $h_{1}=(1+1 / m) h$. Thus, arguing just as in the trivial bundle case, $\omega$ can be viewed as an element in $L_{(0, q+1)}^{2}\left(Q, \mathcal{E}_{1} ; H_{h_{1}(r)}\right)$ and by the above lemma we can produce a $u$ such that $\bar{\partial} \omega=0$ with all the component functions smooth. Continuing to argue as we did earlier in this section we conclude that $u \in \mathcal{E}_{Q}^{\breve{h},(0, q)}(Q)$. Thus the proof of Proposition 5.4 is complete once we establish the proposition above.

We will now start preparations for the proof of Proposition 6.2. For the purposes of the rest of this section we denote, as in [H] , the $\bar{\partial}$-operator on $\mathcal{X}(q)$ by $T$ and the $\bar{\partial}$-operator on $X(q+1)$ by $S$. Then

$$
T: X(q) \cap D(T) \rightarrow X(q+1),
$$


where its domain of definition is

$$
\begin{array}{r}
D(T):=\left\{\left(u_{i k}\right)_{k}=: u_{i k} \in L_{(0, q)}^{2}\left(Q_{i} ; H_{h_{1}(r)}\right) \cap D(\bar{\partial})\right. \\
\left.\left\|\left(\bar{\partial} u_{i k}\right) ; L_{(0, q+1)}^{2}\left(Q_{i} ; H_{h_{1}(r)}\right)\right\|<\infty \forall k\right\} ;
\end{array}
$$

recall that $D(\bar{\partial}) \subset L_{(0, q)}^{2}\left(Q_{i} ; H_{h_{1}(r)}\right)$ is defined as in (6.6). In the same way we have

$$
S: X(q+1) \cap D(S) \rightarrow X(q+2) .
$$

Since the Hilbert spaces here are finite Cartesian products, the next claim follows from the corresponding properties of the component operators.

Lemma 6.3. Both operators $T$ and $S$ are densely defined and closed.

As a consequence, the adjoint operator $T^{*}: X(q+1) \cap D\left(T^{*}\right) \rightarrow X(q)$ and its domain can be defined in the standard way.

Recall that we have written $\mathcal{H}(q)$ for $L_{(0, q)}^{2}\left(Q, \mathcal{E}_{1} ; H_{h_{1}(r)}\right)$. We write $\tilde{T}: \mathcal{H}(q) \cap$ $D(T) \rightarrow \mathcal{H}(q+1)$ for the restriction of $T$ and $\tilde{S}: \mathcal{H}(q+1) \cap D(S) \rightarrow \mathcal{H}(q+2)$ for the restriction of $S$. To analyze the operators $\tilde{T}$ and $\tilde{S}$ let us write

$$
X^{2}(q)=\prod_{i<j} \prod_{k=1}^{n} L_{(0, q)}^{2}\left(Q_{i} \cap Q_{j} ; H_{h_{1}(r)}\right) .
$$

Let us introduce the following notation, $\left(u_{i}, u_{j}\right)$ stands for an entry in $X(q)$ which is non-zero only in positions $i, j$ where the entries are as indicated. We have an exact sequence

$$
0 \rightarrow \mathcal{H}(q) \rightarrow X(q) \stackrel{L}{\rightarrow} X^{2}(q)
$$

where $L$ is given by

$$
\left.L\left(u_{i}, u_{j}\right)\right|_{Q_{i^{\prime}} \cap Q_{j^{\prime}}}= \begin{cases}0 & \text { if }\left(i^{\prime}, j^{\prime}\right) \neq(i, j), \\ u_{j}-G_{j i} u_{i} & \text { if }\left(i^{\prime}, j^{\prime}\right)=(i, j) .\end{cases}
$$

The expression $u_{j}-G_{j i} u_{i}$ is to be interpreted as first restricting $u_{i}$ and $u_{j}$ to $Q_{i} \cap Q_{j}$. The orthogonal complement $\mathcal{H}(q)^{\perp}$ is then given as the image of the adjoint $L^{*}$. An easy calculation shows that

$$
L^{*}\left(u_{i j}\right)=\left(-{ }^{t} \bar{G}_{j i} u_{i j}, u_{i j}\right) .
$$

Were we use same notation as above and we interpret the $u_{i j}$ and the $-{ }^{t} \bar{G}_{j i} u_{i j}$ as elements in $L_{(0, q)}^{2}\left(Q_{j} ; H_{h_{1}(r)}\right)$ and $L_{(0, q)}^{2}\left(Q_{i} ; H_{h_{1}(r)}\right)$ using the inclusions (given by extension by zero) $L_{(0, q)}^{2}\left(Q_{i} \cap Q_{j} ; H_{h_{1}(r)}\right) \subset L_{(0, q)}^{2}\left(Q_{j} ; H_{h_{1}(r)}\right)$ and $L_{(0, q)}^{2}\left(Q_{i} \cap Q_{j} ; H_{h_{1}(r)}\right) \subset$ $L_{(0, q)}^{2}\left(Q_{i} ; H_{h_{1}(r)}\right)$, respectively.

We now have

Lemma 6.4. The spaces $\mathcal{H}(q)$ and $\mathcal{H}(q)^{\perp} \subset \mathcal{X}(q)$ contain dense subspaces $\mathcal{H}^{\infty}(q)$ and $\mathcal{H}^{\infty, \perp}(q)$, respectively, consisting of $C^{\infty}$-functions. 
Proof. The space $\mathcal{H}^{\infty}(q)$ is of course just the space of smooth sections of the bundle $\mathcal{E}_{1}$ and as such it is of course dense in $\mathcal{H}(q)$. As was pointed above the $\mathcal{H}(q)^{\perp}$ is a finite linear combination of elements of the form $L^{*}\left(u_{i j}\right)=\left(-{ }^{t} \bar{G}_{j i} u_{i j}, u_{i j}\right)$. To construct $\mathcal{H}^{\infty, \perp}(q)$ we take linear combinations of the $L^{*}\left(u_{i j}\right)$ where $u_{i j}$ are smooth functions on $Q$ with support in $Q_{i} \cap Q_{j}$. The $L^{*}\left(u_{i j}\right)$ are smooth and $\mathcal{H}^{\infty, \perp}(q)$ is dense by construction.

As the operators $\tilde{T}$ and $\tilde{S}$ are clearly closed, the lemma above immediately implies that

$$
\text { The operators } \tilde{T} \text { and } \tilde{S} \text { are closed and densely defined. }
$$

Proof of Proposition 6.2 In the language we just introduce we are to show that if $\omega$ is in the kernel of $\tilde{S}$ then it is in the image of $\tilde{T}$. We make use of the following well-known lemma, see [H], Lemma 4.1.1], for example,

Lemma 6.5. Let $\mathcal{T}$ be a linear, closed operator from a dense subspace of $H_{1}$ into $\mathrm{H}_{2}$, where $\mathrm{H}_{j}$ are Hilbert spaces, and let $\mathrm{F} \subset \mathrm{H}_{2}$ be a closed subspace such that the range of $\mathcal{T}$ satisfies $R_{\mathcal{T}} \subset F$. Then, $R_{\mathcal{T}}=F$ if and only if $\|g\|_{H_{2}} \leqslant C\left\|\mathcal{T}^{*} g\right\|_{H_{1}}$ for all $g \in F \cap D\left(\mathcal{T}^{*}\right)$.

Applying Lemma 6.1 component-wise implies that the range of the operator $T$ is ker $S$, thus, by Lemma 6.5.

$$
\|g\| \leqslant C\left\|T^{*} g\right\|
$$

for all $g \in \operatorname{ker} S \cap D\left(T^{*}\right)$ for some $C$. Thus, in order to prove Proposition 6.2, it suffices to show that

$$
\|g\| \leqslant C\left\|\tilde{T}^{*} g\right\| \quad \text { for all } g \in \operatorname{ker} \tilde{S} \cap D\left(\tilde{T}^{*}\right) \text { for some } C \text {. }
$$

To do so, in view of (6.2), it suffices to show that $D\left(\tilde{T}^{*}\right)=D\left(T^{*}\right) \cap \mathcal{H}(q+1)$ and that $\tilde{T}^{*}=T^{*}$ on $D\left(\tilde{T}^{*}\right)$. By definition, $D\left(\tilde{T}^{*}\right)$ consists of functions $g \in \mathcal{H}(q+1)$ for which there exists a constant $C=C(g)>0$ such that

$$
\left|(\tilde{T} v \mid g)_{q+1}\right| \leqslant C\|v\|
$$

for all $v \in D(\tilde{T}) \subset \mathcal{H}(q)$. Thus, we are reduced to showing that if $g$ is above then we also have

$$
\left|(T v \mid g)_{q+1}\right| \leqslant C^{\prime}\|v\|
$$

for all $v \in D(T) \subset X(q)$ for some $C^{\prime}$.

Note that, of course, $T$ preserves $\mathcal{H}(q)$, but it does not preserve $\mathcal{H}(q)^{\perp}$. However, as we will show next, it preserves $\mathcal{H}(q)^{\perp}$ up to a bounded operator. Let us now consider $L^{*}\left(u_{i j}\right)=\left(-{ }^{t} \bar{G}_{j i} u_{i j}, u_{i j}\right) \in \mathcal{H}(q)^{\perp}$. Then we see that

$$
\begin{gathered}
T L^{*}\left(u_{i j}\right)=\left(\bar{\partial}\left(-{ }^{t} \bar{G}_{j i} u_{i j}\right), \bar{\partial} u_{i j}\right)=\left(-{ }^{t} \bar{G}_{j i} \bar{\partial} u_{i j}, \bar{\partial} u_{i j}\right)+\left(\left(-\bar{\partial}^{t} \bar{G}_{j i}\right) u_{i j}, 0\right) \\
=L^{*} T\left(u_{i j}\right)+\left(\left(-\bar{\partial}^{t} \bar{G}_{j i}\right) u_{i j}, 0\right)=L^{*} T\left(u_{i j}\right)+R\left(u_{i j}\right) .
\end{gathered}
$$

where $R: X^{2}(q) \rightarrow X(q+1)$ is the bounded operator given by $R\left(u_{i j}\right)=\left(\left(-\bar{\partial}^{t} \bar{G}_{j i}\right) u_{i j}, 0\right)$. 
Let us now pick $w \in \mathcal{H}^{\infty}(q)$ and $w^{\perp} \in \mathcal{H}^{\infty, \perp}(q)$. The elements $v$ of the form $v=$ $w+w^{\perp}$ are dense in $X(q)$. Note that we can assume that $w^{\perp}=L^{*} u_{i j}$ as it is a linear combination of such expressions. Then,

$$
\begin{aligned}
& \left|(T v \mid g)_{q+1}\right|=\left|\left(T\left(w+w^{\perp}\right) \mid g\right)_{q+1}\right| \leqslant\left|(T w \mid g)_{q+1}\right|+\left|\left(T w^{\perp} \mid g\right)_{q+1}\right| \leqslant \\
& C\|w\|+\left|\left(T L^{*} u_{i j} \mid g\right)_{q+1}\right|=C\|w\|+\left|\left(L^{*} T u_{i j} \mid g\right)_{q+1}+\left(R u_{i j} \mid g\right)_{q+1}\right|= \\
& C\|w\|+\left|\left(R u_{i j} \mid g\right)_{q+1}\right| \leqslant C\|w\|+\|R\|\left\|u_{i j}\right\|\|g\| \leqslant(C+\|R\|\|\| g \|)\|v\|,
\end{aligned}
$$

because $w^{\perp}=\left(-{ }^{t} \bar{G}_{j i} u_{i j}, u_{i j}\right)$ and hence $\left\|u_{i j}\right\| \leqslant\left\|w^{\perp}\right\|$. Thus, the linear map $v \mapsto$ $(T v \mid g)_{q+1}$ extends to $D(T)$ as a bounded map and (6.23) holds for all $v \in D(T)$. In other words, $g \in D\left(T^{*}\right)$ and therefore $D\left(\tilde{T}^{*}\right) \subset D\left(T^{*}\right) \cap \mathcal{H}(q+1)$. Also, the identity $\left(v \mid T^{*} g\right)_{q}=\left(v \mid \tilde{T}^{*} g\right)_{q}$ holds for all $v \in D(\tilde{T})$ and $g \in D\left(\tilde{T}^{*}\right)$, and hence $T^{*}=\tilde{T}^{*}$ on $D\left(\tilde{T}^{*}\right)$. This completes the proof of Proposition 6.2 .

\section{TOPOLOGY ON THE SHEAVES AND APPROXIMATION LEMMAS}

In this section we define a topology on global sections of coherent sheaves on compact blocks. We use these topologies to prove approximation lemmas which will be used in the next section.

Let $\mathcal{F}^{\breve{h}}$ be a coherent $\mathcal{A}_{\mathbb{C}^{N}}^{\check{h}}$-module where, as usual, $h$ is a function satisfying condition (5.1). We consider a Stein exhaustion $X_{\nu}$ of $\mathbb{C}^{N}$ by compact blocks, i.e., the $X_{\nu}$, $\nu=1, \ldots$ are compact blocks with $X_{\nu} \subset X_{\mu}$ if $\nu<\mu$ and $\bigcup X_{\nu}=\mathbb{C}^{N}$. We might as well choose the exhaustion to be given as

$$
X_{\nu}=\left\{z=\left(z_{1}, \ldots, z_{N}\right) \in \mathbb{C}^{N}|| \operatorname{Re} z_{i}|\leqslant \nu \quad| \operatorname{Im} z_{i} \mid \leqslant \nu\right\} .
$$

We apply the Cartan A part of theorem 5.1, i.e. the existence of surjections

$$
\left(\mathcal{A}_{X_{\nu}}^{\check{h}}\right)^{\oplus p_{\nu}} \rightarrow \mathcal{F}_{X_{\nu}}^{\check{h}}
$$

to the compact blocks $X_{\nu}$ to obtain presentations

$$
\mathcal{A}_{\mathbb{C}^{N}}^{\check{h}}\left(X_{\nu}\right)^{\oplus p_{\nu}} \rightarrow \mathcal{F}^{\check{h}}\left(X_{\nu}\right) .
$$

We now fix these presentations. Recall that we constructed $\mathcal{F}^{\check{k}}$ in section $\$ 4$ associated to the sheaf $\mathcal{F}^{\breve{h}}$ and a level $k \leqslant h$. By base change the presentations (7.1) then give rise to analogous presentations

$$
\left(\mathcal{A}_{X_{\nu}}^{\check{k}}\right)^{\oplus p_{\nu}} \rightarrow \mathcal{F}_{X_{\nu}}^{\check{k}}
$$

and passing to global sections we have

$$
\mathcal{A}_{\mathbb{C}^{N}}^{\check{k}}\left(X_{\nu}\right)^{\oplus p_{\nu}} \rightarrow \mathcal{F}^{\check{k}}\left(X_{\nu}\right) .
$$

Let us write $k:=h / 2$. The space $\mathcal{F}^{\check{h}}\left(X_{\nu}\right)$ is a direct limit of an increasing sequence of Banach spaces $\left(\mathcal{F}_{\nu, m}^{\breve{h}},\|\cdot\|_{X_{\nu}, m}\right) \subset \mathcal{F}^{\breve{h}}\left(X_{\nu}\right), m \in \mathbb{N}$. To see this we argue as follows. Since $X_{\nu}$ is compact, for any continuous function $h_{1}: X_{\nu} \rightarrow \mathbb{R}_{+}$which satisfies $h_{1}(x)>h(x)$ 
for $x \in X_{\nu}$, we can find $\varepsilon>0$ such that $h_{1} \geqslant(1+\varepsilon) h$. Hence, the space $\mathcal{A}_{\mathbb{C}^{N}}^{\check{h}}\left(X_{\nu}\right)$ is a countable direct limit of Banach spaces,

$$
\mathcal{A}_{\mathbb{C}^{N}}^{\check{h}}\left(X_{\nu}\right)=\underset{h_{1}>h}{\lim _{\longrightarrow}} \mathcal{A}_{\mathbb{C}^{N}}^{h_{1}}\left(X_{\nu}\right)=\underset{m \rightarrow \infty}{\lim _{m \rightarrow \infty}} \mathcal{A}_{\mathbb{C}^{N}}^{(1+1 / m) h}\left(X_{\nu}\right)
$$

where the norm of $\mathcal{A}_{\mathbb{C}^{N}}^{(1+1 / m) h}\left(X_{\nu}\right)$ is given by

$$
\sup _{X_{\nu}}\|f\|_{\left(1+\frac{1}{m}\right) h}=\sup _{x \in X_{\nu}}\|f(x)\|_{\left(1+\frac{1}{m}\right) h(x)}=\sup _{x \in X_{\nu}} \sum_{j=0}^{\infty}\left|a_{j}(x)\right|\left\|t^{j}\right\|_{(1+1 / m) h(x)} .
$$

for $f(x, t)=\sum_{j=0}^{\infty} a_{j}(x) t^{j}$ (cf. (3.1) ). Then, $\left(\mathcal{A}_{\mathbb{C}^{N}}^{\breve{h}}\left(X_{\nu}\right)\right)^{\oplus p_{\nu}}$ and thus also $\mathcal{F}\left(X_{\nu}\right)$ are countable direct limits of Banach spaces, the latter as a quotient in the presentations we fixed in (7.2)

$$
\left(\mathcal{A}_{\mathbb{C}^{N}}^{\check{h}}\left(X_{\nu}\right)\right)^{\oplus p_{\nu}} \rightarrow \mathcal{F}\left(X_{\nu}\right) .
$$

Now, $\mathcal{A}_{\mathbb{C}^{N}}^{\breve{k}}\left(X_{\nu}\right)$ has a bounded set which contains a neighborhood of 0 of $\mathcal{A}_{\mathbb{C}^{N}}^{\breve{h}}\left(X_{\nu}\right)$. Indeed, from (7.5) it is easy to see that the bounded set can, for example, be chosen to be $\left\{f: \sup _{X_{\nu}}\|f\|_{4 / 3 k}=\sup _{X_{\nu}}\|f\|_{2 / 3 h} \leqslant 1\right\}$, which contains the neighborhood

$$
\Gamma\left(\bigcup_{m \in \mathbb{N}}\left\{f: \sup _{X_{\nu}}\|f\|_{(1+1 / m) h} \leqslant 1\right\}\right) ;
$$

here $\Gamma$ denotes the absolutely convex hull.

Let us recall that by (4.4) we have an inclusion $\mathcal{F}^{\breve{h}}\left(X_{\nu}\right) \subset \mathcal{F}^{\breve{k}}\left(X_{\nu}\right)$. Furthermore:

Lemma 7.1. Given $\nu$, there is a bounded set $B(\nu) \subset \mathcal{F}^{\breve{k}}\left(X_{\nu}\right)$ which contains a neighborhood $U(\nu)$ of 0 of $\mathcal{F}^{\check{h}}\left(X_{\nu}\right) \subset \mathcal{F}^{\check{k}}\left(X_{\nu}\right)$.

Proof. By the remark just above, the space $\left(\mathcal{A}_{\mathbb{C}^{N}}^{\check{k}}\left(X_{\nu}\right)\right)^{\oplus p_{\nu}}$ has a bounded set which is a neighborhood of 0 of $\left(\mathcal{A}_{X}^{\check{h}}\left(X_{\nu}\right)\right)^{\oplus p_{\nu}}$. The claim follows from the commutative diagram

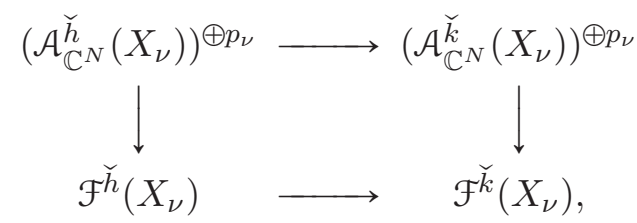

where the horizontal mappings are inclusions and the vertical ones are continuous surjections defining the topologies of the spaces on the last row.

We denote the Minkowski functional of the bounded set $B(\nu)$ by

$$
\|f\|_{B(\nu)}=\inf \{r>0: f \in r B(\nu)\} .
$$

This is well defined for $f \in \mathcal{F}^{\breve{h}}\left(X_{\nu}\right)$, by the lemma above. Moreover:

$$
\begin{gathered}
\text { convergence with respect to }\|\cdot\|_{B(\nu)} \text { implies } \\
\text { convergence in the topology of } \mathcal{F}^{\breve{k}}\left(X_{\nu}\right) \text {. }
\end{gathered}
$$


We will now formulate the second approximation theorem we will make use of in the next section. In this lemma we fix the level $h$ but compare the topologies on various $\mathcal{F}^{\breve{h}}\left(X_{\nu}\right)$.

To compare these norms for various $X_{\nu}$ let us consider $X_{\nu}$ and $X_{\nu+1}$. By restricting the presentation

$$
\left(\mathcal{A}_{X_{\nu+1}}^{\check{h}}\right)^{\oplus p_{\nu+1}} \rightarrow \mathcal{F}_{X_{\nu+1}}^{\check{h}}
$$

to $X_{\nu}$ we obtain two presentations of $\left.\mathcal{F}^{\check{h}}\right|_{X_{\nu}}$ and we then choose $s_{\nu+1, \nu}$ once and for all so that

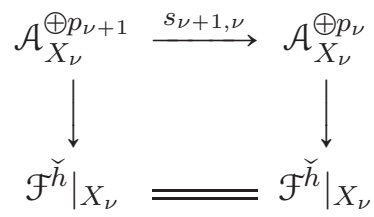

commutes. Passing to global sections we obtain:

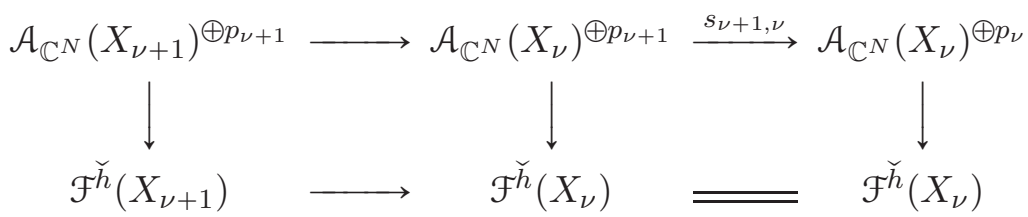

with surjective columns.

Lemma 7.2. Given $\nu, \gamma_{\nu}^{\prime} \in \mathcal{F}\left(X_{\nu}\right)$ and a collection of continuous seminorms $\|\cdot\|_{\nu, n}$ on $\mathcal{F}\left(X_{n}\right), n \leqslant \nu$, then, for any $\varepsilon>0$, there exists $\gamma_{\nu} \in \mathcal{F}^{\breve{h}}\left(X_{\nu+1}\right)$ such that for all $n \leqslant \nu$ we have

$$
\left\|\left.\gamma_{\nu}\right|_{X_{n}}-\left.\gamma_{\nu}^{\prime}\right|_{X_{n}}\right\|_{\nu, n} \leqslant \varepsilon
$$

Proof. $1^{\circ}$. We show that the restriction map $\mathcal{A}_{\mathbb{C}^{N}}^{\check{h}}\left(X_{\nu+1}\right) \rightarrow \mathcal{A}_{\mathbb{C}^{N}}^{\check{h}}\left(X_{\nu}\right)$ has dense image. Given $g_{\nu}^{\prime} \in \mathcal{A}_{\mathbb{C}^{N}}^{\check{h}}\left(X_{\nu}\right)$ we write $g_{\nu}^{\prime}(x)=\sum_{j=0}^{\infty} a_{\nu, j}(x) t^{j}$, where $a_{\nu, j}$ are scalar holomorphic mappings on $X_{\nu}$. We also choose $m$ such that $g_{\nu}^{\prime} \in \mathcal{A}_{X}^{(1+1 / m) h}\left(X_{\nu}\right)$ (see (17.4)), i.e., we have $g_{\nu}^{\prime}(x) \in A_{(1+1 / m) h(x)}$ for all $x \in X_{\nu}$.

Given $\varepsilon$ we now choose $l$ such that (cf. (7.5))

$$
\sup _{x \in X_{n}}\left\|\sum_{j=l}^{\infty} a_{\nu, j}(x) t^{j}\right\|_{(1+1 / m) h(x)}=\sup _{x \in X_{n}} \sum_{j=l}^{\infty}\left|a_{\nu, j}(x)\right|\left\|t^{j}\right\|_{(1+1 / m) h(x)}<\varepsilon / 2
$$

for all $n \leqslant \nu$. Then, we have finitely many scalar holomorphic mappings $a_{\nu, j}: X_{\nu} \rightarrow \mathbb{C}$, $j<l$, and using the Runge approximation theorem on $X_{\nu}$, we approximate all of them by corresponding polynomials $P_{\nu, j}: X \rightarrow \mathbb{C}, j<k$ such that

$$
\sup _{x \in X_{n}} \sum_{j=0}^{k-1}\left|P_{\nu, j}(x)-a_{\nu, j}(x)\right|\left\|t^{j}\right\|_{(1+1 / m) h(x)}<\varepsilon / 2
$$


for all $n \leqslant \nu$. We define $g_{\nu} \in \mathcal{A}_{\mathbb{C}^{N}}^{\check{h}}\left(X_{\nu+1}\right)$ by

$$
g_{\nu}(x)=\sum_{j=0}^{k-1} P_{\nu, j}(x) t^{j} \quad, \quad x \in X_{\nu+1} .
$$

It is clear from above that for all $n \leqslant \nu$

$$
\sup _{x \in X_{n}}\left\|g_{\nu}(x)-g_{\nu}^{\prime}(x)\right\|_{(1+1 / m) h(x)}<\varepsilon .
$$

$2^{\circ}$. We now consider the following commutative diagram:

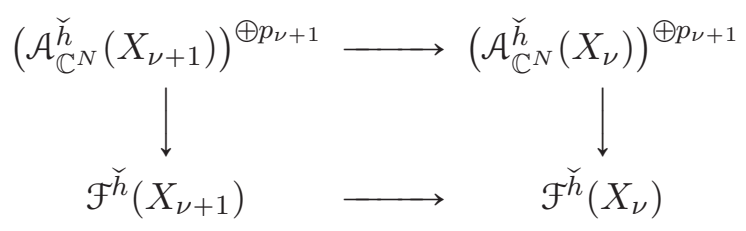

By the above argument, the top mapping has dense image. Also, the vertical mappings in (7.10) are continuous surjections. Hence, the bottom map necessarily also has dense image. Moreover, the expression

$$
\|f\|_{\nu}=\sum_{n=1}^{\nu}\left\|\left.f\right|_{X_{n}}\right\|_{\nu, n}
$$

is a continuous seminorm on $\mathcal{F}^{\check{h}}\left(X_{\nu}\right)$, since the restriction maps are continuous. Consequently, we can approximate any element of $\mathcal{F}^{\breve{h}}\left(X_{\nu}\right)$ arbitrarily well by an element of $\mathcal{F}^{\breve{h}}\left(X_{\nu+1}\right)$ with respect to the seminorm (7.11). Hence, (7.9) follows.

\section{Cartan theorems}

In this section we prove Cartan's theorems A and B in our setting. As we mentioned in the introduction, we follow the classical strategy and pass from the compact case of the theorem to the general case by a Stein exhaustion. However, in our setting this process is not as straightforward as in the classical case as controlling various seminorms is more tricky. To control these norms we are forced to allow the auxiliary base rings $A_{\breve{h}}$ to vary along $X$.

We state our main theorem here for completeness.

Theorem 8.1. Let $X$ be a Stein manifold and let $\mathcal{F}$ be a coherent $\mathcal{A}_{X}$-module. Then $\mathrm{H}^{i}(X, \mathcal{F})=0$ for $i \geqslant 1$. Furthermore, the sheaf $\mathcal{F}$ is generated by its global sections.

Remark 8.2. We can slightly generalize the theorem above. It also holds for coherent $\mathcal{A}_{X}^{\breve{h}}$-module provided that $h$ satisfies condition (5.1). The proof below goes through in this case with minor adjustments.

As we remarked earlier we can assume that $X=\mathbb{C}^{N}$ as any Stein manifold can be embedded in $\mathbb{C}^{N}$ for some $N$. We first remark that theorem A follows formally from theorem $\mathrm{B}$ in a similar manner as as was explained in section 5 for compact blocks. In our setting the argument there gives a surjection $\mathrm{H}^{0}(X, \mathcal{F}) \otimes_{A} \mathcal{A}_{X} \rightarrow \mathcal{F}$. 
We now consider a Stein exhaustion $\left(X_{\nu}\right)_{\nu \in \mathbb{N}}$ by compact compact blocks in $\mathbb{C}^{N}$, as in the previous section. By theorem 5.1 we can conclude that

$$
\mathrm{H}^{i}\left(X_{\nu}, \mathcal{F}\right)=0 \text { for } i \geqslant 1 .
$$

By a very general argument, see, for example, GrRe, Chapter 4, $\S 1$, Theorem 4] we conclude that $\mathrm{H}^{i}(X, \mathcal{F})=0$ for $i \geqslant 2$. Thus we are left to deal with the case $i=1$.

To prove the vanishing of $\mathrm{H}^{1}(X, \mathcal{F})$ we fix a countable cover of $\mathbb{C}^{N}$ by precompact open Stein domains $U_{i}, i \in \mathbb{N}$. We can and will assume that the cover has the property that for any $X_{\nu}$ only finitely many of the $U_{i}$ have a non-trivial intersection with $X_{\nu}$. We will next choose Stein domains $\left(V_{i}\right)_{i \in \mathbb{N}}$ such that $V_{i} \subset \bar{V}_{i} \subset U_{i}$ and such that the $V_{i}$ still form a cover of $\mathbb{C}^{N}$. O course, the sets $\bar{V}_{i}$ are compact. We also assume to be given a representative $\alpha \in \prod \mathcal{F}\left(U_{i} \cap U_{j}\right)$ of a class in $\mathrm{H}^{1}(X, \mathcal{F})$; we fix $\alpha$ for the rest of the proof. Let us now consider the Chech complexes:

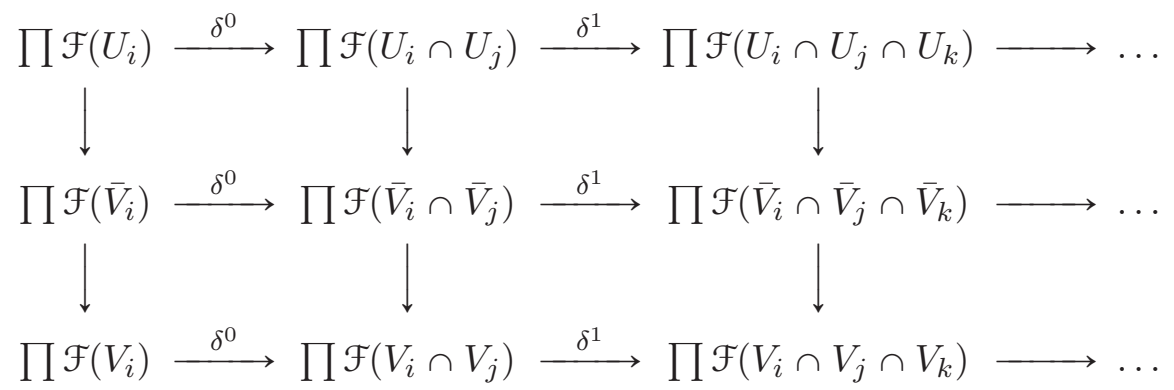

Now, the first row and the third row both compute the cohomology $\mathrm{H}^{*}(X, \mathcal{F})$ and the restriction map from the first row to the third row induces the identity map on $\mathrm{H}^{*}(X, \mathcal{F})$. Restricting the cocycle $\alpha$ to the cover $\left(V_{i}\right)$ we obtain $\alpha_{V} \in \prod \mathcal{F}\left(V_{i} \cap V_{j}\right)$. As $\alpha_{V}$ comes from $\prod \mathcal{F}\left(\bar{V}_{i} \cap \bar{V}_{j}\right)$ we see that each component $\alpha_{V}(i, j) \in \mathcal{F}^{h_{i, j}}\left(V_{i} \cap V_{j}\right)$ for some constants $h_{i, j}>0$.

Let us choose a twice differentiable function $h: X \rightarrow \mathbb{R}^{+}$(recall that $h$ is a function of the norm $r=|x|$, only) such that it is smaller than the above constants $h_{i, j}$ on the sets $U_{i} \cap U_{j}$ and satisfies the condition (5.1). This is possible because for each $X_{\nu}$ there are only finitely many $V_{i}$ intersecting $X_{\nu}$. Let us write $h_{\nu}$ for the minimum of the $h_{i, j}$ arising from the $V_{i}$ which intersect $X_{\nu}$. Thus, the function $h: \mathbb{R}^{+} \rightarrow \mathbb{R}^{+}$has to satisfy:

$$
h(r)<h_{\nu} \quad \text { if } \nu-1 \leqslant r \leqslant \nu .
$$

To obtain an $h$ satisfying this condition one simply chooses $H$ to decrease sufficiently rapidly and then $h$ is given by formula (5.2).

We also denote $k=h / 2$ as in Lemma 7.1. As a consequence, we have

$$
\alpha_{V} \in \prod_{i, j} \mathscr{F}^{\check{h}}\left(V_{i} \cap V_{j}\right)
$$

where $\mathcal{F}^{\breve{h}}$ is the sheaf associated to $\mathcal{F}$ constructed in section 4 . According to the discussion in $\$$, see in particular (4.4), we have canonical inclusions $\mathcal{F}^{\check{h}} \subset \mathcal{F}^{\check{k}} \subset \mathcal{F}$. 

is a

$$
\beta_{\nu}^{\prime} \in \prod_{i \in I} \mathcal{F}^{\check{h}}\left(U_{i} \cap X_{\nu}\right)
$$

such that

$$
\left(\delta^{0} \mid X_{\nu}\right) \beta_{\nu}^{\prime}=\left.\alpha\right|_{X_{\nu}}
$$

We have:

Lemma 8.3. Given $\alpha \in \operatorname{Ker} \delta^{1} \subset \prod \mathcal{F}^{\check{h}}\left(V_{i} \cap V_{j}\right)$ as in (8.1), there exist sequences $\left(\beta_{\nu}\right)_{\nu=1}^{\infty}, \beta_{\nu} \in \prod_{i} \mathcal{F}^{\breve{h}}\left(V_{i} \cap X_{\nu}\right)$, and $\left(\delta_{\nu}\right)_{\nu=1}^{\infty}, \delta_{\nu} \in \prod_{i} \mathcal{F}^{\breve{k}}\left(X_{\nu-1}\right)$ with the following properties for all $\nu$ :

1) $\left(\left.\delta^{0}\right|_{X_{\nu}}\right) \beta_{\nu}=\left.\alpha\right|_{X_{\nu}}$

2) $\left.\left(\beta_{\nu+1}+\delta_{\nu+1}\right)\right|_{X_{\nu-1}}=\left.\left(\beta_{\nu}+\delta_{\nu}\right)\right|_{X_{\nu-1}}$ in $\prod_{i} \mathcal{F}^{\breve{k}}\left(V_{i} \cap X_{\nu-1}\right)$

Let us first argue that this lemma implies the main result. By property 2) of the lemma, there exists a section $\beta \in \prod_{i} \mathcal{F}^{\breve{k}}\left(V_{i}\right) \subset \prod_{i} \mathcal{F}\left(V_{i}\right)$ such that $\left.\beta\right|_{X_{\nu}}=\left(\beta_{\nu+1}+\right.$ $\left.\delta_{\nu+1}\right)\left.\right|_{X_{\nu}}$ for all $\nu$. Property 1) of the lemma then implies

$$
\left(\left.\delta^{0}\right|_{X_{\nu}}\right) \beta=\left(\left.\delta^{0}\right|_{X_{\nu}}\right)\left(\left.\beta_{\nu+1}\right|_{X_{\nu}}\right)+\left(\left.\delta^{0}\right|_{X_{\nu}}\right)\left(\left.\delta_{\nu+1}\right|_{X_{\nu}}\right)=\left.\alpha\right|_{X_{\nu}}
$$

which gives $\delta^{0}(\beta)=\alpha$. As $\alpha$ was an arbitrary 1-cocycle we conclude that $\mathrm{H}^{1}(X, \mathcal{F})=0$. Thus, it remains to prove the lemma.

Proof of lemma 8.3. We make the following definition by induction: assume that $\nu \in \mathbb{N}$ and that

$$
\beta_{m} \in \prod_{i} \mathcal{F}^{\check{h}}\left(U_{i} \cap X_{m}\right), m \leqslant \nu
$$

have been chosen such that

$$
\left(\left.\delta^{0}\right|_{X_{m}}\right) \beta_{m}=\left.\alpha\right|_{X_{m}} \text { for all } m=1, \ldots, \nu .
$$

We define

$$
\gamma_{\nu}^{\prime}=\left.\beta_{\nu+1}^{\prime}\right|_{X_{\nu}}-\beta_{\nu}
$$

hence, we have

$$
\left(\left.\delta^{0}\right|_{X_{\nu}}\right) \gamma_{\nu}^{\prime}=\left(\left.\delta^{0}\right|_{X_{\nu}}\right)\left(\left.\beta_{\nu+1}^{\prime}\right|_{X_{\nu}}\right)-\left(\left.\delta^{0}\right|_{X_{\nu}}\right) \beta_{\nu}=\left.\alpha\right|_{X_{\nu}}-\left.\alpha\right|_{X_{\nu}}=0
$$

by (8.3) and (8.5); as a consequence, $\gamma_{\nu}^{\prime} \in \mathcal{F}^{\check{h}}\left(X_{\nu}\right)$.

We now apply Lemma 7.1 for all $n \leqslant \nu$. Thus we obtain bounded sets $B(n) \subset \mathcal{F}^{\breve{k}}\left(X_{n}\right)$ which contain open neighborhoods $U(n)$ of the origin in $\mathcal{F}^{\breve{h}}\left(X_{n}\right)$. We also write $\|\cdot\|_{\nu, n}$ for the continuous seminorms on $\mathcal{F}^{\breve{h}}\left(X_{n}\right)$ whose unit ball is the open neighborhood $U(n)$. By Lemma 7.1 and the notation introduced in (7.6) we can find constants $K(\nu, n)>1$ such that

$$
\|f\|_{B(n)} \leqslant K(\nu, n)\|f\|_{\nu, n}
$$


for all $f \in \mathcal{F}^{\breve{h}}\left(X_{n}\right)$. We now apply Lemma 7.2 to $\gamma_{\nu}^{\prime}$ and thus find $\gamma_{\nu} \in \mathcal{F}^{\breve{h}}\left(X_{\nu+1}\right)$ such that

$$
\left\|\left.\gamma_{\nu}^{\prime}\right|_{X_{n}}-\left.\gamma_{\nu}\right|_{X_{n}}\right\|_{\nu, n} \leqslant 2^{-\nu}\left(\max _{n \leqslant \nu} K(\nu, n)\right)^{-1} .
$$

To complete the induction step we define

$$
\beta_{\nu+1}=\beta_{\nu+1}^{\prime}-\gamma_{\nu} \in \prod_{i} \mathcal{F}^{\check{h}}\left(U_{i} \cap X_{\nu+1}\right),
$$

which implies

$$
\left(\left.\delta^{0}\right|_{X_{\nu+1}}\right) \beta_{\nu+1}=\left(\left.\delta^{0}\right|_{X_{\nu+1}}\right) \beta_{\nu+1}^{\prime}-\left(\left.\delta^{0}\right|_{X_{\nu+1}}\right) \gamma_{\nu}=\left.\alpha\right|_{X_{\nu+1}}-0 .
$$

It remains to construct the $\delta_{\nu}$. For all $\nu \in \mathbb{N}$ we now define

$$
s_{j}^{(\nu)}=\left.\beta_{\nu+j}\right|_{X_{\nu}}-\beta_{\nu}, j=1,2, \ldots
$$

We claim that the sequence $\left(s_{j}^{(\nu)} \mid X_{\nu}\right)_{j=1}^{\infty}$, viewed as a sequence in $\mathcal{F}^{\breve{k}}\left(X_{\nu}\right)$ converges with respect to the seminorm $\|\cdot\|_{B(\nu)}$, when $j \rightarrow \infty$. Thus, by (7.7), the sequence $\left(s_{j}^{(\nu)} \mid X_{\nu}\right)_{j=1}^{\infty}$ converges in $\mathcal{F}^{\breve{k}}\left(X_{\nu}\right)$.

We will then set

$$
\delta_{\nu}=\lim _{j \rightarrow \infty} s_{j}^{(\nu)} .
$$

To verify the claim, let $\nu$ be fixed. We first observe that by (8.6), (8.9),

$$
\left.\beta_{n+1}\right|_{X_{n}}-\beta_{n}=\gamma_{n}^{\prime}-\left.\gamma_{n}\right|_{X_{n}},
$$

for all $n \leqslant \nu$, hence,

$$
\begin{aligned}
s_{j}^{(\nu)} & =\left.\beta_{j+\nu}\right|_{X_{\nu}}-\left.\beta_{j+\nu-1}\right|_{X_{\nu}}+\left.\beta_{j+\nu-1}\right|_{X_{\nu}}-\ldots+\left.\beta_{\nu+1}\right|_{X_{\nu+1}}-\left.\beta_{\nu}\right|_{X_{\nu}} \\
& =\left.\sum_{k=0}^{j} \gamma_{\nu+k-1}^{\prime}\right|_{X_{\nu}}-\left.\gamma_{\nu+k-1}\right|_{X_{\nu}} \in \mathcal{F}^{\check{h}}\left(X_{\nu}\right) .
\end{aligned}
$$

In the same way,

$$
\begin{aligned}
& s_{j}^{(\nu)}-s_{l}^{(\nu)} \\
= & \left.\beta_{j+\nu}\right|_{X_{\nu}}-\left.\beta_{j+\nu-1}\right|_{X_{\nu}}+\left.\beta_{j+\nu-1}\right|_{X_{\nu}}-\ldots-\left.\beta_{l+\nu}\right|_{X_{\nu}} \\
= & \left.\sum_{k=l}^{j-1} \gamma_{k+\nu}^{\prime}\right|_{X_{\nu}}-\left.\gamma_{k+\nu}\right|_{X_{\nu}},
\end{aligned}
$$

hence, by (8.7), (8.8),

$$
\begin{aligned}
& \left\|s_{j}^{(\nu)}-s_{l}^{(\nu)}\right\|_{B(\nu)} \\
\leqslant & \sum_{k=l}^{j-1} K(k+\nu, \nu)\left\|\left.\gamma_{k+\nu}^{\prime}\right|_{X_{\nu}}-\left.\gamma_{k+\nu}\right|_{X_{\nu}}\right\|_{k+\nu, \nu} \\
\leqslant & \sum_{k=l}^{j-1} 2^{-\nu-k}<\varepsilon
\end{aligned}
$$


if $l$ is large enough. Therefore the $s_{j}^{(\nu)}$ form a Cauchy sequence in $\mathcal{F}^{\breve{k}}\left(X_{\nu}\right)$ and so we have constructed the $\delta_{\nu} \in \mathcal{F}^{\breve{k}}\left(X_{\nu}\right)$.

Now, from (8.10), (8.11) we deduce that the sequences $\left(\left.\beta_{\nu+j}\right|_{X_{\nu}}\right)_{j \in \mathbb{N}}$ also converge in $\mathcal{F}^{\breve{k}}\left(X_{\nu}\right)$ and hence,

$$
\begin{aligned}
& \left.\left(\delta_{\nu}-\delta_{\nu+1}\right)\right|_{X_{\nu-1}}=\left.\lim _{j \rightarrow \infty}\left(s_{j}^{(\nu)}-s_{j}^{(\nu+1)}\right)\right|_{X_{\nu-1}} \\
= & -\left.\beta_{\nu}\right|_{X_{\nu-1}}+\left.\beta_{\nu+1}\right|_{X_{\nu-1}}+\left.\lim _{j \rightarrow \infty} \beta_{\nu+j}\right|_{X_{\nu-1}}-\left.\lim _{j \rightarrow \infty} \beta_{\nu+1+j}\right|_{X_{\nu-1}} \\
= & -\left.\beta_{\nu}\right|_{X_{\nu-1}}+\left.\beta_{\nu+1}\right|_{X_{\nu-1}} .
\end{aligned}
$$

\section{REFERENCES}

[Bu1] Lutz Bungart, Holomorphic functions with values in locally convex spaces and applications to integral formulas, Trans. Amer. Math. Soc. 1111964 317-344.

[FG] J. Frisch and J. Guenot, Prolongement de faisceaux analytiques cohérents, Invent. Math. 7 1969 321-343.

[GrRe] Grauert, Hans; Remmert, Reinhold Coherent analytic sheaves, Grundlehren der Mathematischen Wissenschaften 265. Springer-Verlag, Berlin, 1984. xviii+249 pp.

[Gr] Grothendieck, Alexandre,Sur certains espaces de fonctions holomorphes. I. (French) J. Reine Angew. Math. 192, (1953) 35-64.

[H] Hörmander, L., An introduction to Complex analysis in several variables, 3rd Ed. NorthHolland.

[KaVi] Masaki Kashiwara and Kari Vilonen, Microdifferential systems and the codimension-three conjecture, Ann. of Math. (2) 180-2 2014 573-620.

[Ko] Gottfried Köthe, Topological vector spaces. II. Grundlehren der Mathematischen Wissenschaften, 237. Springer-Verlag, New York-Berlin, 1979. xii+331 pp.

[Kr] Steven Krantz, Function theory of several complex variables, 2. ed. AMS Chelsea Publishing, Providence, (2001).

[P] Albrecht Pietsch, Nuclear locally convex spaces, Ergebnisse der Mathematik und ihrer Grenzgebiete, Band 66. Springer-Verlag, New York-Heidelberg, (1972).

[Sch] Pierre Schapira, Microdifferential Systems in the Complex Domain, Grundlehren der Mathematischen Wissenschaften 269 Springer-Verlag, Berlin (1985).

[Se] Serre, Jean-Pierre Géométrie algébrique et géométrie analytique, Ann. Inst. Fourier, Grenoble 6 (1955-1956), 1-42.

[Siu] Yum-tong Siu, Extending coherent analytic sheaves, Ann. of Math. (2) 901969108 -143.

[T] Günther Trautmann, Ein Kontinuittssatz für die Fortsetzung kohärenter analytischer Garben, Arch. Math. (Basel) $181967188-196$.

Department of Mathematics and Statistics, University of Helsinki, P.O.Box 68, Fi00014 Helsinki, Finland

E-mail address: jari.taskinen@helsinki.fi

School of Mathematics and Statistics, University of Melbourne, ViC 3010, Australia, and Department of Mathematics and Statistics, University of Helsinki, P.O.Box 68, FI00014 Helsinki, Finland

E-mail address: kari.vilonen@unimelb.edu.au and kari.vilonen@helsinki.fi 